\title{
Peptidylarginine deiminases 2 and 4 modulate innate and adaptive immune responses in TLR-7-dependent lupus
}

\author{
Yudong Liu, ${ }^{1}$ Yaíma L. Lightfoot, ${ }^{1}$ Nickie Seto, ${ }^{1}$ Carmelo Carmona-Rivera, ${ }^{1}$ Erica Moore, ${ }^{1}$ \\ Rishi Goel,, Liam O'Neil, ${ }^{1}$ Pragnesh Mistry, ${ }^{1}$ Victoria Hoffmann, ${ }^{2}$ Santanu Mondal, ${ }^{3}$ \\ Padmavathy Nandha Premnath, ${ }^{3}$ Katherine Gribbons, ${ }^{1}$ Stefania Dell'Orso, ${ }^{4}$ Kan Jiang, ${ }^{4}$ \\ Paul R. Thompson, ${ }^{3}$ Hong-Wei Sun, ${ }^{4}$ Scott A. Coonrod, ${ }^{5}$ and Mariana J. Kaplan ${ }^{1}$ \\ 1Systemic Autoimmunity Branch, Intramural Research Program (IRP), National Institute of Arthritis and Musculoskeletal \\ and Skin Diseases (NIAMS), and 'Division of Veterinary Resources, Office of the Director, NIH, Bethesda, Maryland, USA. \\ ${ }^{3}$ University of Massachusetts School of Medicine, Worcester, Massachusetts, USA. ${ }^{4}$ Biodata Mining and Discovery Section, \\ Office of Science and Technology, IRP, NIAMS/ NIH, Bethesda, Maryland, USA. ${ }^{5}$ Baker Institute for Animal Health, College \\ of Veterinary Medicine, Cornell University, Ithaca, New York, USA.
}

The peptidylarginine deiminases PAD2 and PAD4 are implicated in the pathogenesis of several autoimmune diseases. PAD4 may be pathogenic in systemic lupus erythematosus (SLE) through its role in neutrophil extracellular trap (NET) formation that promotes autoantigen externalization, immune dysregulation, and organ damage. The role of this enzyme in mouse models of autoimmunity remains unclear, as pan-PAD chemical inhibitors improve clinical phenotype, whereas PAD4-KO models have given conflicting results. The role of PAD2 in SLE has not been investigated. The differential roles of PAD2 and PAD4 in TLR-7-dependent lupus autoimmunity were examined. Padi4 ${ }^{-/}$displayed decreased autoantibodies, type I IFN responses, immune cell activation, vascular dysfunction, and NET immunogenicity. Padi2 ${ }^{-/-}$mice showed abrogation of Th subset polarization, with some disease manifestations reduced compared with WT but to a lesser extent than $\mathrm{Padi}^{-/-}$mice. RNA sequencing analysis revealed distinct modulation of immunerelated pathways in PAD-KO lymphoid organs. Human T cells express both PADs and, when exposed to either PAD2 or PAD4 inhibitors, displayed abrogation of Th1 polarization. These results suggest that targeting PAD2 and/or PAD4 activity modulates dysregulated TLR-7-dependent immune responses in lupus through differential effects of innate and adaptive immunity. Compounds that target PADs may have potential therapeutic roles in T cell-mediated diseases.

Conflict of Interest: PRT holds a patent of AFM30a and was the founder of the scientific advisory board for Padlock Therapeutics, which was acquired by Bristol Myers Squibb in April of 2016.

Authorship note: YL, YLL, and NS are co-first authors.

License: Copyright 2018, American Society for Clinical Investigation.

Submitted: September 12, 2018 Accepted: November 1, 2018 Published: December 6, 2018

Reference information: JCI Insight. 2018;3(23):e124729. https://doi.org/10.1172/jci. insight.124729.

\section{Introduction}

Systemic lupus erythematosus (SLE) is a systemic autoimmune syndrome characterized by profound immune dysregulation affecting innate and adaptive immunity. SLE manifests with pleiotropic clinical manifestations, generation of autoantibodies against nuclear antigens, and the development of immune complexes (ICs) that stimulate type I IFN production by plasmacytoid DCs (pDCs) (1). Type I IFNs play important roles in the development, progression, and clinical manifestations of SLE (2). In SLE, there is also a striking increase in the development of cardiovascular (CV) complications due to accelerated atherosclerosis, which represents an important cause of morbidity and mortality (3). Significant abnormalities in myeloid cells, pDCs, and lymphocytes are detected in human SLE and in murine models of the disease $(1,4)$. Therefore, identifying putative pharmacologic targets that decrease autoantigen generation and modulate immune dysregulation in SLE is needed. While several available therapies are effective in mitigating SLE manifestations, many of them are associated with significant side effects due to a lack of specificity, while other more targeted treatments may not address all the pathogenic immune features that contribute to this complex syndrome.

Protein citrullination is the process by which the basic amino acid residue arginine is converted into the neutral residue citrulline (5). This reaction is catalyzed by a family of calcium-dependent enzymes called peptidylarginine deiminases (PADs), of which there are 5 isozymes (PAD1-4 and PAD6) encoded by distinct genes in the human genome (5). These isozymes have unique tissue localization and overlapping substrates, 
and there is $70 \%-95 \%$ homology in their amino acid sequence among mammals. PADs are involved in various important physiological processes, including immune responses, cell signaling, and gene expression regulation. Abnormal protein citrullination and/or aberrant immune responses to citrullinated antigens have been implicated in the pathogenesis of various cancers and autoimmune and neurological diseases (6-9).

Intracellular citrullination may occur as part of the physiological function of PADs and has been best described for PAD4. PAD4 appears to be the main member of the PAD family with a nuclear localization signal, and its enzymatic activity impacts gene expression by directly citrullinating transcription factors or by regulating chromatin structure through histone citrullination $(10,11)$. PAD4 is expressed at high levels in neutrophils, and histone citrullination mediated by PAD4 has been implicated in the formation of neutrophil extracellular traps (NETs) following certain types of stimulation (12-14). NET formation is a mechanism of neutrophil cell death whereby, following a complex and still incompletely characterized intracellular pathway, these cells extrude a meshwork of nuclear material bound to granule proteins to the extracellular space (15). While this process is considered an important antimicrobial strategy, dysregulation in NET formation has recently been implicated in the pathogenesis of systemic autoimmune diseases including rheumatoid arthritis (RA) (7, 16, 17), psoriasis (18), systemic vasculitis (19), and SLE (20-22). An imbalance of NET formation and degradation occurs in SLE, and this phenomenon may play a role in augmenting the half-life of externalized modified autoantigens that, in a predisposed host, would contribute to fueling the immune system, which would promote type I IFN responses, inflammasome activation, aberrant adaptive immunity, and tissue damage, including vascular injury (20,21, 23-25). In addition to the role in NET formation, PAD4 enhances the activity of NF- $\mathrm{kB}$ in neutrophils, independently of changes in histone citrullination, with potential therapeutic implications for TLR-mediated inflammation (26).

$\mathrm{PAD} 2$ is the other PAD isozyme that is expressed at high levels in some immune cell subsets, primarily monocytes and macrophages, but is also described in neutrophils $(27,28)$. However, the role of PAD2 in autoimmune and inflammatory diseases in general, and SLE in particular, is less clear. In specific models of arthritis, PAD2 is not required for NET formation but can potentially modify immune responses by citrullinating cytokines like TNF- $\alpha$ (29). Recent studies suggest that PADs may potentially impact T cell biology, as PAD4-KO mouse T cells lose citrullinated histone H3 (Cit-H3) staining upon activation. Furthermore, PAD2- and PAD4-mediated citrullination can modulate Th2 and Th17 cytokine expression (30).

These observations indicate that PAD2 and PAD4 may play important and distinct roles in the modulation of aberrant immune responses in autoimmune conditions. The roles of PAD2 and PAD4 in SLE pathogenesis, however, remain unclear, primarily because of conflicting results in various murine models. Systemic administration of chemical inhibitors targeting both PAD2 and PAD4 can significantly abrogate lupus phenotype and associated immune dysregulation and organ damage in 2 genetically prone mouse models of lupus, NZM2328 and MRL/lpr $(31,32)$. These compounds can also modulate vasculopathy and thrombosis in lupus and atherosclerosis models $(31,33)$. In contrast, MRL/lpr mice that genetically lack PAD4 are not protected from disease (34), while pristane-induced lupus is exacerbated in PAD4-KO mice (35). The reasons for these discrepancies are not clear and may be related to the dual inhibition of PAD2 and PAD4 with the chemical inhibitors, off-target effects of the inhibitors, the intricacies of the specific genetic models, putative antiinflammatory effects of PADs (36), differences in microbiome, or the impact of partial inhibition versus complete inhibition of PAD activity. It has also been observed that redundancy exists in the effect of the PADs on various cellular functions, and how PADs regulate each other remains unclear (30). These observations support that mouse models of lupus, similar to human disease, are heterogeneous and driven by different pathways and that understanding the roles of the PADs in various aspects of immune dysregulation and organ damage is crucial in assessing whether targeting these enzymes represents a viable option in the treatment of autoimmune diseases.

In SLE, the dosage of the endosomal TLR-7 has been proposed to be crucial in disease pathogenesis in humans and mice. TLR-7 overexpression induces spontaneous lupus-like disease, while suppressing signaling through this TLR in lupus-prone mice hampers disease development (37-40). Given that the role of PAD2 in SLE animal models has not been addressed and that the exact role of PAD4 inhibition remains to be further elucidated, we investigated the role of these 2 isozymes in an induced model of TLR-7-dependent lupus-like autoimmunity in the absence of confounding murine autoimmunity susceptibility genes. We found differential effects of PAD2 and PAD4 on the induction of immune dysregulation, tissue damage, endothelial dysfunction, immune cell gene expression profiles, and autoimmunity features. We also describe how these PADs modulate NET immunogenicity and $\mathrm{T}$ cell responses, and we demonstrate that selective inhibitors of PAD2 and PAD4 also affect human T cell function. 


\section{Results}

PAD2 and PAD4 differentially regulate TLR-7-mediated lupus autoimmunity. To examine the differential role of different PADs in SLE progression independently of contributions by murine lupus susceptibility genes, a previously described inducible model of TLR-7-dependent lupus-like disease was used (41). WT, Padi2 ${ }^{-1-}$, and Padi4 $^{-1-}$ mice (all in FVB background) were exposed epicutaneously to the TLR-7 agonist imiquimod for 6 weeks, 3 times a week, as described (41). During the third week of treatment, the mice were exposed to a subacute dose of ultraviolet B ray-narrowband (UVB-narrowband) radiation for 5 consecutive days. Although no weight loss developed at the experimental endpoint, imiquimod-treated WT — and, to a lesser extent, imiquimod-treated $\mathrm{Padi2}^{-/-}$mice but not $\mathrm{Padi}^{-{ }^{--}}$mice - showed signs of physical discomfort and reduced grooming. Furthermore, treated WT and $\mathrm{Padi}^{-/-}$mice displayed significant increases in spleen size and spleen weight when compared with untreated mice. In contrast, spleen size was significantly lower in the imiquimod-treated Padi4 ${ }^{-1}$ mice when compared with WT-treated mice (Figure 1A). While quantification of serum anti-dsDNA, a lupus-specific autoantibody, revealed that imiquimod-treated mice - in all 3 groups - had significantly higher antibody titers than untreated mice (Figure 1B), levels of these antibodies were approximately 2-fold higher in WT FVB when compared with Padi2 ${ }^{-/-}$and Padi4 ${ }^{-/-}$mice (Figure 1B). When additional lupus autoantibodies were measured, anti-ribonucleoprotein/Smith antigen (anti-RNP/Sm) serum levels did not differ across the 3 groups, while anti-histone antibodies were significantly decreased in Padi4 ${ }^{-/}$and there was a trend for lower levels in the $\mathrm{Padi2}^{-/-}$when compared with WT mice $(P=0.079$, Supplemental Figure 1 ; supplemental material available online with this article; https:// doi.org/10.1172/jci.insight.124729DS1). In contrast, no differences were observed in total serum IgG levels (Figure 1C). These results indicate expansion of specific autoreactive antibody-producing cells in FVB mice upon exposure to a TLR-7 agonist and modulation of these responses by PADs. Nonsignificant alterations in frequency of circulating immune cells were observed to varying degrees in imiquimod-treated WT and Padi2 ${ }^{-/-}$mice, including increased lymphocyte, monocyte, and neutrophil frequencies, but not in Padi4 ${ }^{-/}$mice (Supplemental Figure 2, A-C). Gene expression analyses of splenocytes demonstrated enhanced upregulation of type I IFN-regulated genes in imiquimod-treated FVB but not in treated Padi2 ${ }^{-1}$ or Padi4 ${ }^{-/-}$mice (Figure 1D). Overall, while topical imiquimod administration resulted in significant systemic inflammation and lupus-like features in WT FVB mice, similar to what has been reported for other nonautoimmune mouse strains (41), these features were significantly attenuated in Padi4 $4^{-1-}$ mice and — to a somewhat lesser extent - in Padi2 ${ }^{-/-}$mice.

PADs modulate kidney IC deposition in TLR-7-mediated lupus autoimmunity. In contrast to a previous report (41), administration of imiquimod to FVB mice did not result in significant albuminuria (Supplemental Figure 2D) or in significant changes in the renal histopathologic score (data not shown). However, immunofluorescence analysis of kidney sections revealed moderate to severe $\mathrm{C} 3$ and IgG deposition in the glomeruli of imiquimod-treated WT FVB mice (Figure 2, A and B). While glomerular IC deposition was only slightly reduced in treated $\mathrm{Padi2}^{--}$mice, the $\mathrm{Padi4}^{-1-}$ mice exposed to imiquimod were significantly protected from glomerular IgG deposition when compared with both WT and Padi2 $2^{-1}$ mice (Figure 2, A and B). These results suggest that immune mechanisms controlled by PAD4 activity — and, to a lesser extent, PAD2 either directly or indirectly promote renal IC deposition in response to proinflammatory stimuli.

Padi4 ${ }^{--}$, but not Padi2 ${ }^{-/}$, mice are protected from endothelial dysfunction in TLR-7-mediated lupus autoimmunity. Endothelial dysfunction and vascular damage are common features of murine and human SLE and contribute to heightened morbidity and mortality in this disease (3). To test whether topical imiquimod administration triggers vasomotor dysfunction in these strains, we measured acetylcholine-dependent (Ach-dependent) vasorelaxation responses in thoracic aortas from untreated and imiquimod-treated WT FVB, Padi2 ${ }^{-1-}$, and $\mathrm{Padi4}^{-/}$mice. Treated WT FVB and Padi2 ${ }^{-/-}$mice displayed significantly impaired endothelium-dependent vasorelaxation compared with untreated mice. In contrast, Padi4 ${ }^{-1-}$ mice were protected from developing endothelial dysfunction by imiquimod (Figure 3). These data implicate PAD4-related pathways in endothelial dysfunction characteristic of lupus autoimmunity.

Padi4 ${ }^{-1-}$ NETs decreased vasculopathic and immunostimulatory effects. We and others have demonstrated that NETs can induce and amplify pathogenic type I IFN responses and promote vascular damage in SLE and atherosclerosis models $(20,33,42,43)$. Given the putative role of PADs in NET formation (13), we sought to determine whether the differences observed in disease progression among the different mouse groups were due to differential effects on the ability of neutrophils to form NETs in the presence or absence of PADs. While Padi4 ${ }^{-1}$ neutrophils produced fewer NETs in response to the ionophore A223817, NETs were 

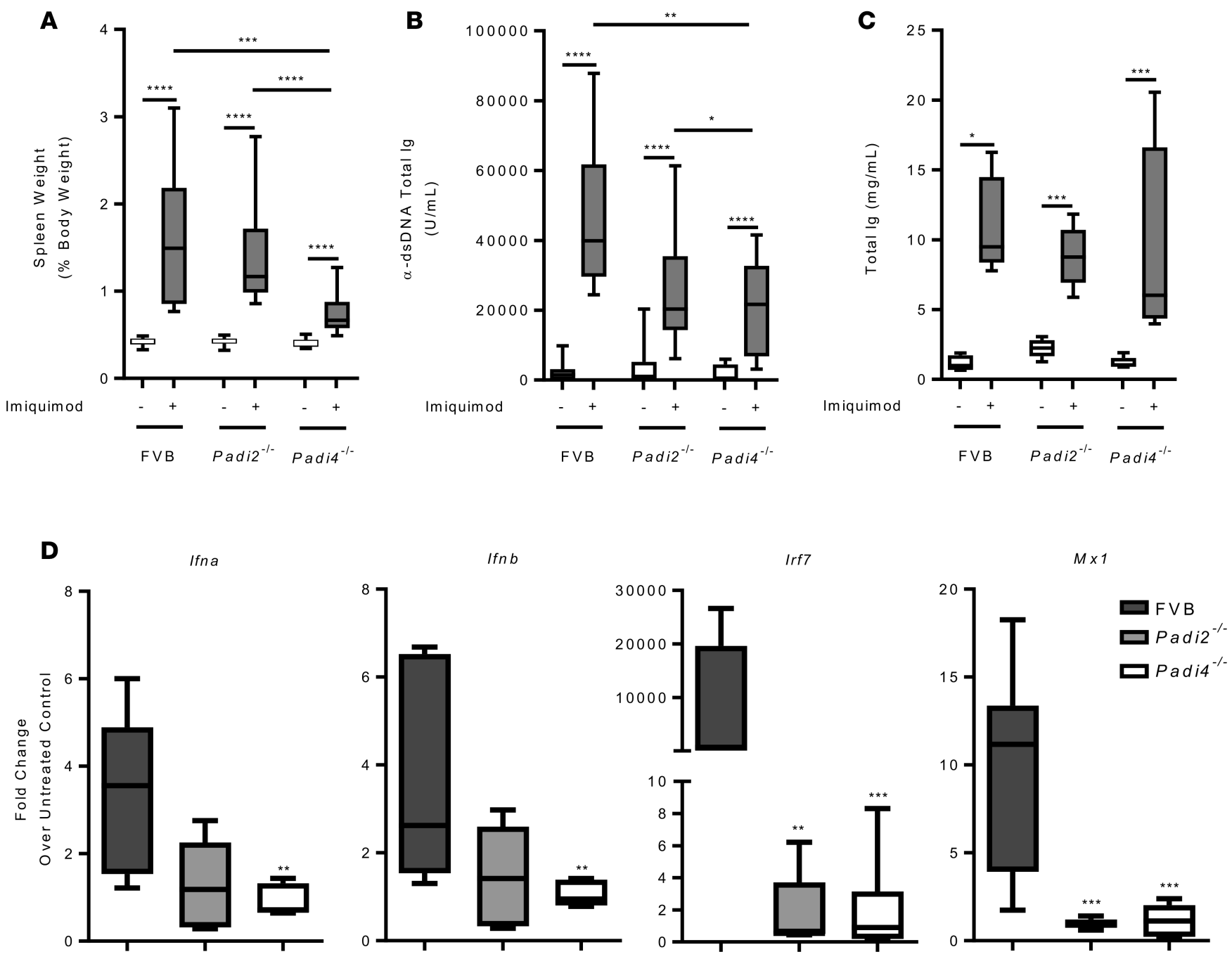

Figure 1. Padi2 and Padi4 deficiencies differentially modulate TLR-7-induced autoimmunity. (A) Quantification of splenomegaly assessed by spleen

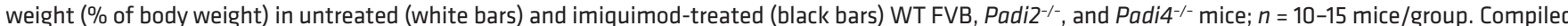
data from 3 independent experiments. (B and C) Levels of circulating anti-dsDNA autoantibodies (B) and total lgG (C) in untreated (white whisker plots) and imiquimod-treated (black whisker plots) FVB, Padi2 ${ }^{-/}$, and Padi4 ${ }^{-/-}$mice. $n=9-15$ mice/group. Compiled data from 3 independent experiments. (D) Gene expression data of splenocytes from treated FVB, Padi2 ${ }^{-/}$, and Padi4 ${ }^{-/-}$mice normalized to gene expression in the corresponding control mice. $n=7$ mice/ group. Compiled data from 2 independent experiments. Box-and-whisker plots show median, lower and upper quartiles, and minimum and maximum values. ${ }^{*} P<0.05,{ }^{* *} P<0.005$, ${ }^{* *} P<0.0005$, ${ }^{* * *} P<0.0001$. Statistical analysis was performed by 2 -tailed Mann-Whitney $U$ test. IMQ , imiquimod.

still present in these mice when compared with stimulated WT FVB and Padi2-- neutrophils (Figure 4). However, Cit-H3 was undetectable in NETs from Padit ${ }^{-/}$mice, while other essential NET components such as DNA and myeloperoxidase (MPO) were detected. Of note, glycolysis, the main metabolic pathway utilized by neutrophils, was not impaired in Padi4 ${ }^{-1}$ neutrophils compared with WT FVB and Padi2 $2^{-1}$ neutrophils (Supplemental Figure 3), suggesting that their reduced capacity to form NETs is not due to an inability to generate ATP required for NET formation. Previous studies indicated that PAD2 may have the ability to localize to the nucleus and citrullinate histones (44). However, Padi2 ${ }^{-/-}$neutrophils did not display impairment in their ability to form NETs, and there were no reductions in Cit-H3 (Figure 4).

To mimic the in vivo setting in subsequent in vitro studies, we stimulated WT FVB, Padi2 ${ }^{-/}$, and Padi4 ${ }^{-1}$ neutrophils with imiquimod to assess NET induction. Protein analysis of imiquimod-induced NETs confirmed that PAD4 is the main isoform responsible for $\mathrm{Cit}-\mathrm{H} 3$ generation in mouse neutrophils (Figure 4C). Since NETs still formed in Padi2 $2^{--}$, and to lesser extent in Padi4 ${ }^{--}$, we examined whether the vasculoprotec-

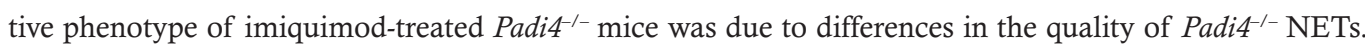
WT FVB aortic rings were exposed to equal concentrations of imiquimod-generated NETs purified from 
A

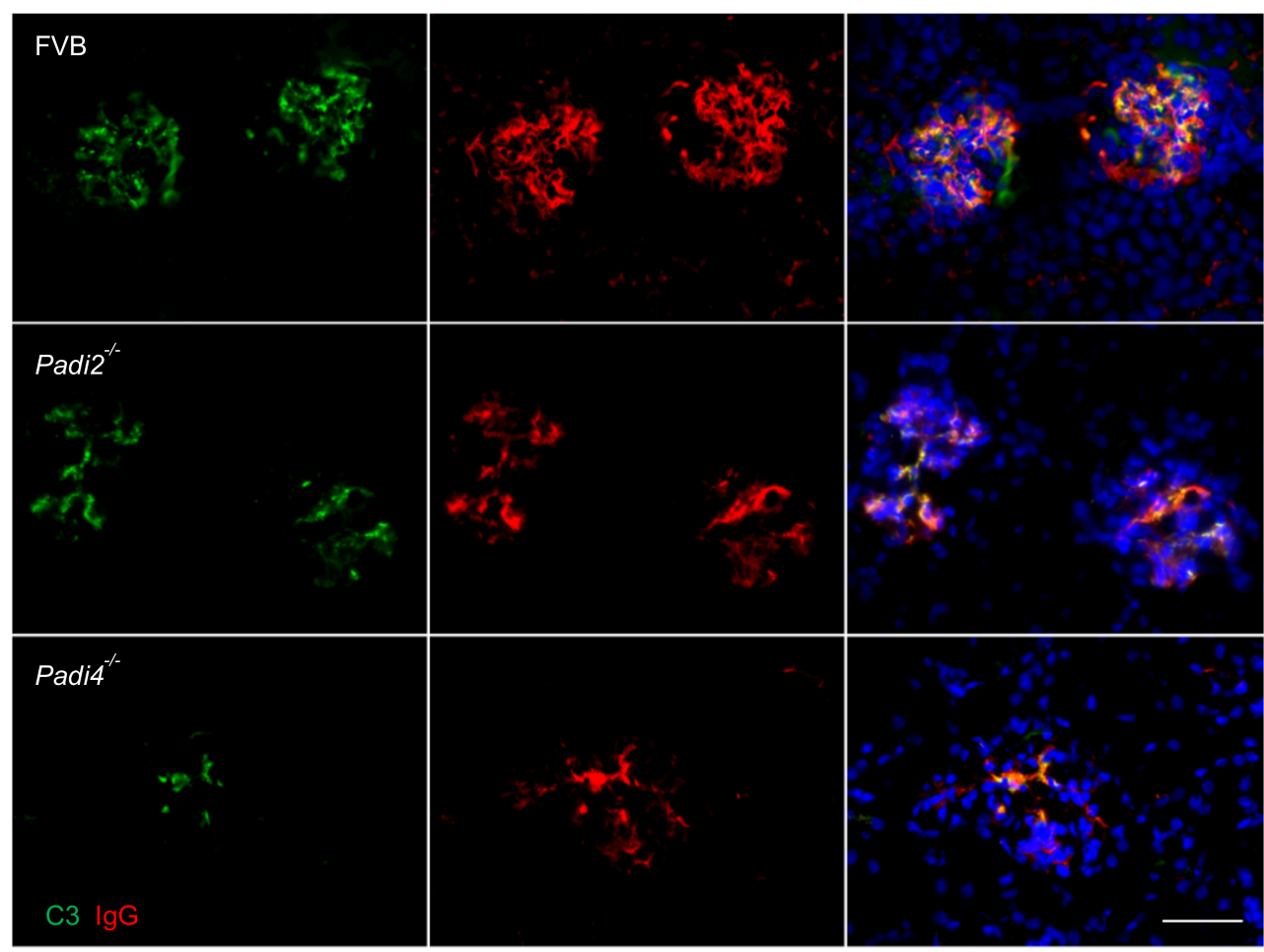

B
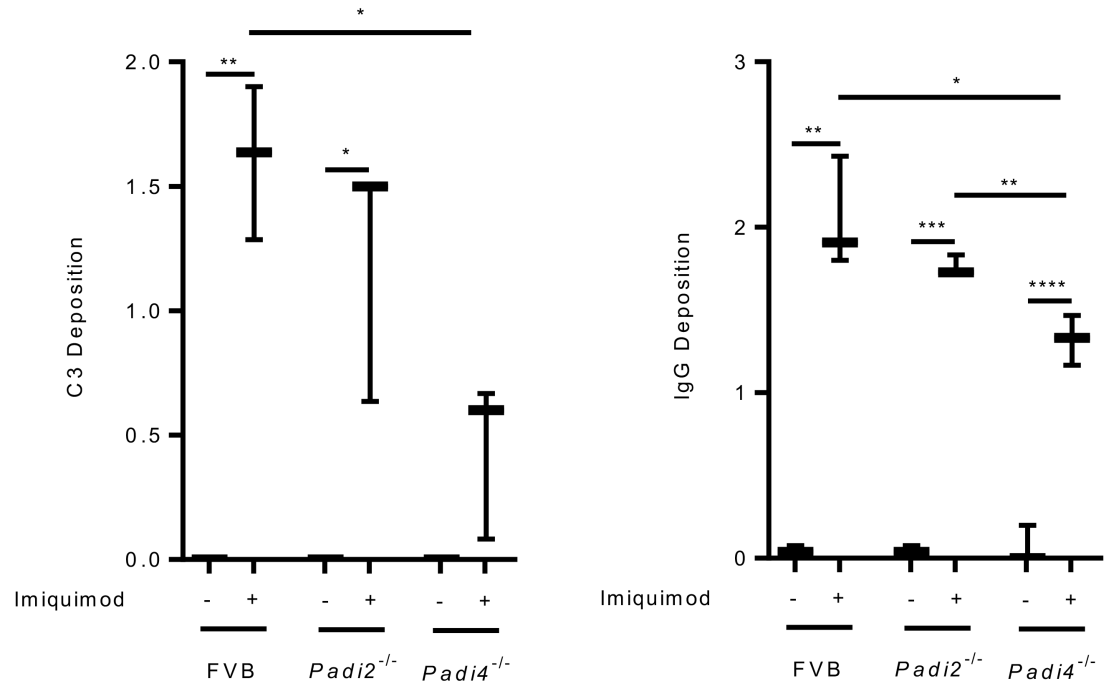

Figure 2. Renal immune complex deposition is attenuated in imiquimod-treated Padi4 ${ }^{-/}$mice. (A and B) Frozen kidney sections were stained for immune complex deposition $(\mathbf{A})$ and quantified based on fluorescence intensity and staining pattern (B); $n=3$ mice/group. Box-and-whisker plots show median, lower and upper quartiles, and minimum and maximum score values. ${ }^{*} P<0.05,{ }^{* *} P<0.005,{ }^{* *} P<0.0005$, ${ }^{* * *} P<0.0001$. Statistical analysis was performed by 2-tailed unpaired $t$ test. Green represents C3, red represents IgG, and blue represents DAPI. The figures on the right are merged images. Total magnification is $40 x$.

neutrophils from the 3 different strains. Similar to the in vivo observations, WT FVB and Padi2 ${ }^{-1-}$ NETs directly impaired endothelium-dependent vasorelaxation, whereas equal amounts of Padi4 ${ }^{-1}$ NETs did not (Figure 4D). To assess whether the quality of the NETs generated by Padi2 ${ }^{--}$or Padi4 ${ }^{--}$would impact the ability of these NETs to induce type I IFN responses in target cells, we incubated FVB mouse splenocytes with imiquimod-induced NETs from WT, Padi2 $2^{--}$, and Padi4 ${ }^{--}$mice and quantified induction of type I IFN-regulated genes (Figure 4E). FVB NETs led to the strongest induction of these genes when compared with Padi2 ${ }^{-1}$ and Padi4 ${ }^{--}$NETs (Figure 4E). Overall, these data indicate that specific modifications of the 
A
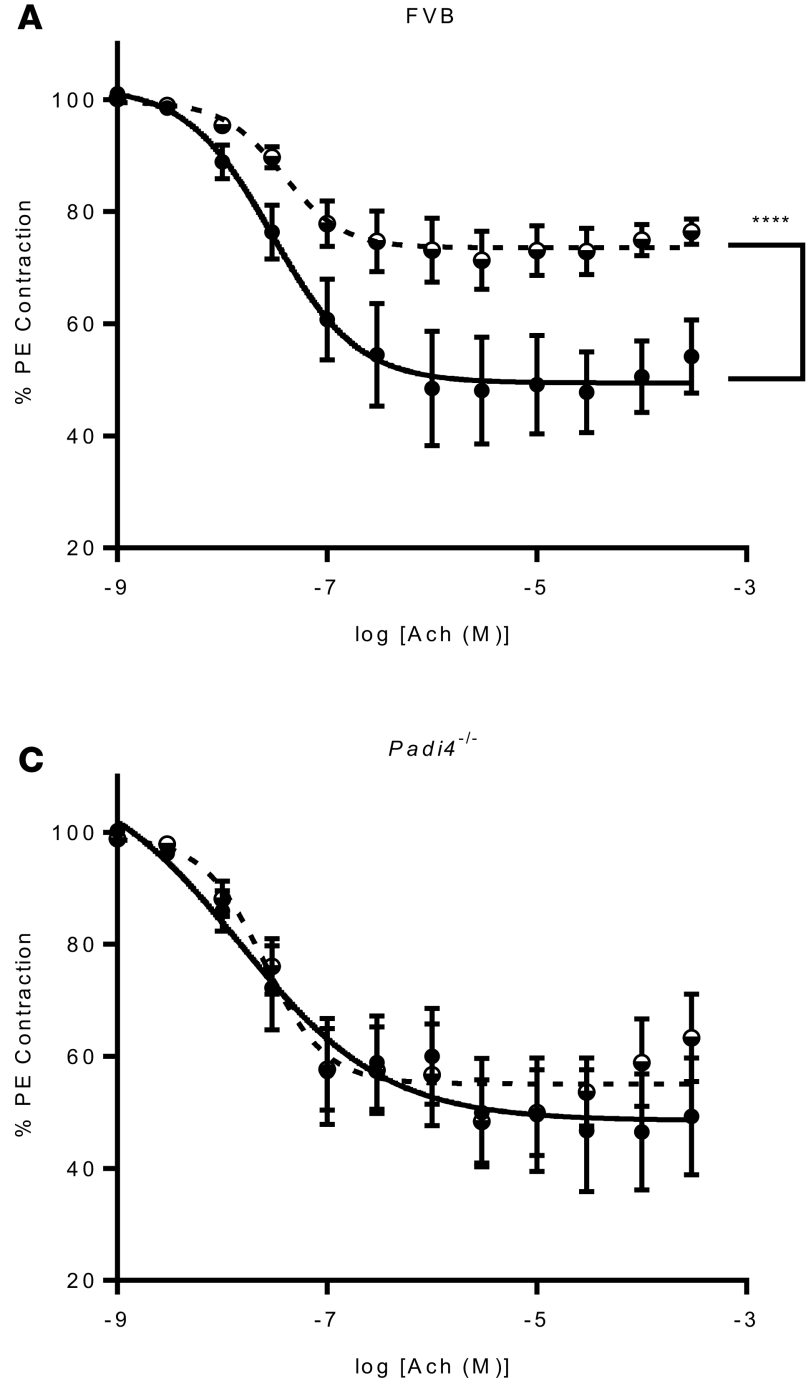

B

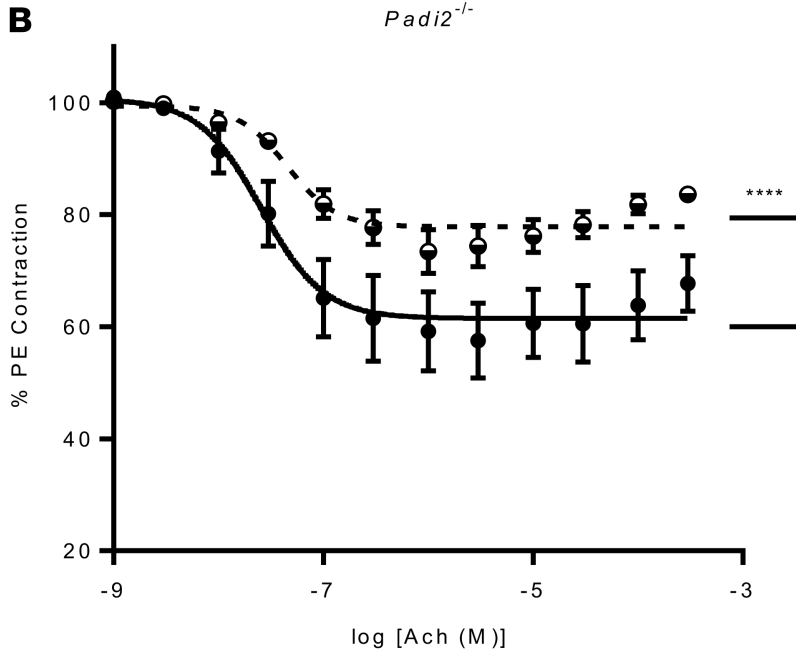

$\log [A c h(M)]$

- - IMQ

Figure 3. Imiquimod-treated Padi4 ${ }^{-/-}$mice retain normal vasorelaxation capacity. (A-C) Aortic rings were isolated from untreated and imiquimod-treated FVB (A), Padi2 ${ }^{-/-}$(B), and Padi4 ${ }^{--}$(C) mice. Acetylcholine-dependent relaxation following phenylephrine $(P E)$ precontraction was determined. $n=6-10$ mice/group. Results represent mean \pm SEM \% vasorelaxation; ${ }^{* * *} P<0.0001$ A 2-way ANOVA with post-hoc Tukey's test was used to compare differences between the groups.

content of the NETs may regulate the degree of immune cell stimulation and endothelial dysfunction mediated by these structures and that only PAD4 influences the vasculopathic effects of NETs, whereas both PAD4 and PAD2 modulate the interferogenic properties of NETs.

Differential secondary lymphoid organ gene regulation in $\mathrm{Padi} 2^{--}$and $\mathrm{Padi4}^{--}$mice in response to TLR-7 activation. At euthanasia, RNA sequencing (RNA-Seq) analysis was performed in spleens and inguinal lymph nodes from imiquimod-treated mice. Gene expression was compared between WT, Padi2 ${ }^{-1-}$, and Padi4 ${ }^{-1}$ mice (Supplemental Tables 1-4). For Padi2 $2^{--}, 2,241$ genes were significantly modified in the lymph nodes compared with WT, including 1,714 that were transcriptionally repressed and 527 that were upregulated. A total of 957 genes were modified in the lymph nodes of $\mathrm{Padi}^{-1}$ mice when compared with WT. Among those, 529 were downregulated and 428 were upregulated when compared with WT. Only $8.9 \%$ of the downregulated genes and 7.2\% of the upregulated genes overlapped between Padi2 ${ }^{-1-}$ and Padi4 ${ }^{-1-}$ mice (Figure 5, A and B). A total of 2,126 genes were differentially regulated between $\mathrm{Padi2}^{-/}$and $\mathrm{Padi}^{-1-}$ lymph nodes. The analysis of spleens by RNA-Seq revealed that 1,913 genes were transcriptionally repressed and 467 were upregulated in Padi2 ${ }^{-/-}$compared with WT, while 2,221 were repressed and 1,178 were upregulated in Padi4 ${ }^{-1}$ compared with WT. The degree of overlap of differentially regulated genes in imiquimod-treated Padi2 ${ }^{-1}$ and Padi4 ${ }^{-1}$ mice, when compared with imiquimod-treated WT, was significantly higher for spleen than for lymph nodes, with $37.3 \%$ of overlap in downregulated genes and $15 \%$ of overlap in upregulated genes (Supplemental Figure 5). Furthermore, 2,288 genes were differentially regulated in Padi2 ${ }^{-/}$compared with Padi4 $^{-1}$ spleens.

Several relevant immune pathways were significantly highlighted in the lymph node pathway analysis (Supplemental Table 3). Pathways associated to type I and type II IFN signaling were significantly 


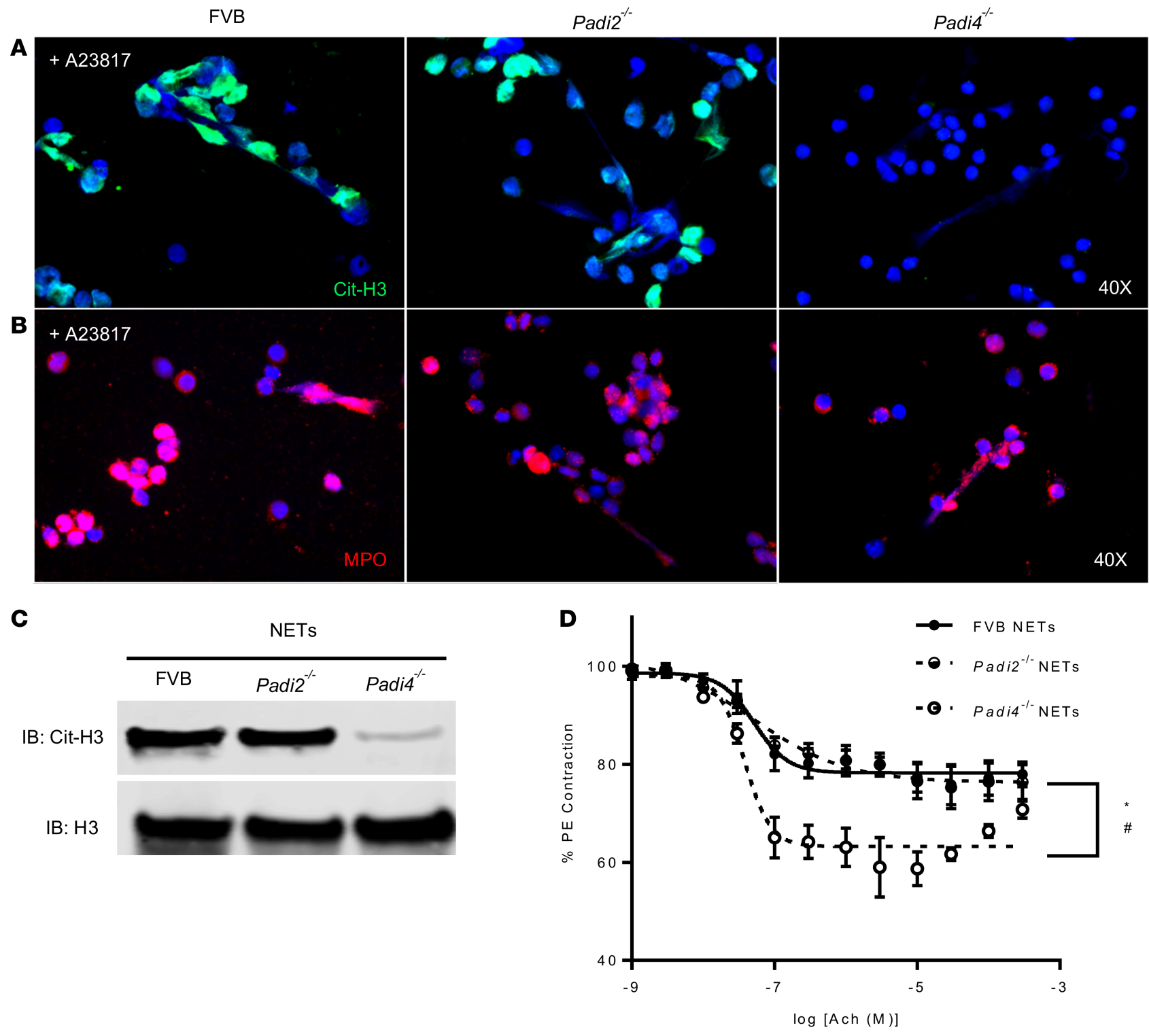

Padi2 $^{-1-}$

Padi4 $^{-1-}$

E
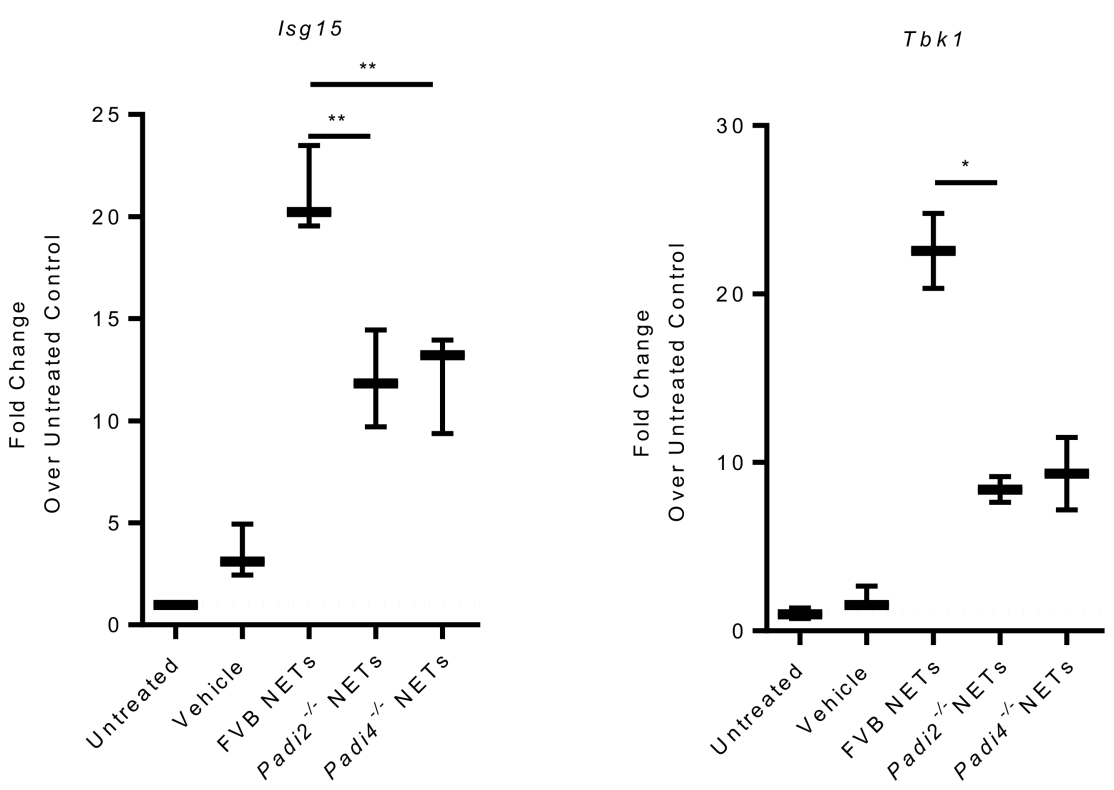
Figure 4. Padi4 ${ }^{-/-}$neutrophils generate NETs lacking citrullinated Histone 3 (Cit-H3) and do not promote vascular damage. (A and B) BM neutrophils of naive FVB, Padi2 $2^{--}$, and Padi4 ${ }^{-/-}$mice were stimulated with the calcium ionophore A23817 for 2 hours to induce NET formation and stained for Cit-H3 (A) and MPO (B). Total magnification is 40x. (C) Lack of Cit-H3 in Padi4 $4^{-/-}$NETs was confirmed by Western blot of imiquimod-induced NETs. Results are representative of 3 experiments (D). Aorta rings were cultured in the presence of imiquimod-induced NETs prior to assessment of vasorelaxation capacity. Results represent $\%$ mean \pm SEM vasorelaxation, $n=3$ mice/group. (E) Splenocytes from naive FVB, Padi2 ${ }^{-1-}$, and Padi4 $4^{--}$mice ( $n=3 /$ group) were cultured for 24 hours in the presence of imiquimod-induced NETs, and the expression of type 1 IFN-inducible genes was analyzed by RT-PCR. ${ }^{*} P<0.05,{ }^{*} P<0.005$. Statistical analysis was performed with 2-tailed unpaired $t$ test. For $\mathbf{D}$, analysis was by 2 -way ANOVA with post-hoc Tukey's test to compare differences between the groups; ${ }^{*} P<$ 0.05 for PAD4 KO vs. FVB; ${ }^{*} P<0.05$ for PAD4 KO vs. PAD2 KO.

downregulated in Padi2 ${ }^{--}$compared with WT, while the upregulated pathways included leukocyte and neutrophil migration, as well as chemotaxis. In contrast, there were no significant canonical pathways differentially downregulated in the Padi4 ${ }^{-/}$lymph nodes compared with WT following in vivo imiquimod exposure. Among the pathways upregulated in the Padi4 ${ }^{-/-}$compared with WT, those related to angiogenesis and epithelial cell proliferation were significantly changed (Supplemental Figure 4 and Supplemental Table 3), while pathways differentially regulated between Padi2 $^{-1-}$ and $\mathrm{Padi4}^{-1-}$ mice included neutrophil migration, chemotaxis, and activation ( downregulated in $\mathrm{Padi4}^{--}$), as well as protein folding and RNA splicing (downregulated in $\mathrm{Padi2}^{-/}$) (Figure 5).

Genes downregulated in the Padi2 $2^{-1}$ lymph nodes compared with WT lymph nodes were enriched with 19 transcription factors with a binding site in the promoter of genes differentially regulated (Supplemental Table 5). Several of these transcription factors, including Irf7, Stat1, Irf1, Irf9, and Irf8, are key transcriptional regulators of type I IFN-dependent immune responses and/or type I IFN signaling. Irf7 was the most significantly downregulated transcription factor in $\mathrm{Padi}^{-/-}$lymph nodes. With regard to Padi4 ${ }^{-/}$lymph nodes, 8 transcription factors had a binding site in the promoter of genes differentially regulated when compare with WT, including upregulation of Gata2, Cebpd, Foxf1, and Hoxd8 and dowregulation of Fhl2. In contrast to what was observed in Padi2 ${ }^{-/}$, these transcription factors target a significantly lower number of molecules (Supplemental Table 6). These results indicate that PAD2 is associated with more robust effects on gene transcription in lymph nodes when compared with PAD4 and that the pathways regulated in this lymphoid organ significantly differ between PAD2 and PAD4. These results further support that the protective effects observed in TLR-7-induced autoimmunity in the PAD2- and PAD4-deficient models are mediated by different pathways.

The pathway analysis of the genes differentially regulated by lack of Padi2 ${ }^{-1-}$ or Padi4 ${ }^{-1}$ in the spleen of imiquimod-treated mice revealed a different profile when compared with findings in lymph nodes (Supplemental Figure 5 and Supplemental Tables 2 and 4). For Padi2 $2^{-1-}$ mice, the top downregulated pathways included ribonucleoprotein complex biogenesis and RNA processing and splicing, while the top upregulated pathways were associated to autophagy and organelle disassembly (Supplemental Figure 5 and Supplemental Table 4). In Padi4 ${ }^{-1}$ mice, the top downregulated pathways similarly included RNA processing and ribonucleoprotein biogenesis, as well as DNA replication, while upregulated pathways were associated to T cell activation and differentiation (Supplemental Figure 5 and Supplemental Table 4). When comparing Padi2 ${ }^{-/-}$and $\mathrm{Padi4}^{-/}$, the top downregulated pathways in $\mathrm{Padi}^{-/-}$were those related to cytoplasmic translation and intracellular transport across organelles, ER stress, and glycoprotein metabolic processes, while downregulated pathways in the Padi4 $^{-/}$included autophagy and organelle disassembly pathways (Supplemental Figure 5).

The differentially expressed genes in the Padi2 ${ }^{-1}$ spleens compared with WT spleens were enriched with 13 transcription factors with a binding site in the promoter of genes differentially regulated (Supplemental Table 7). Among them, Mycn (downregulated), Crem (downregulated), and Klf3 (upregulated) accounted for the ability to interact with a large number of genes differentially regulated in the RNA-Seq analysis. In Padi4 ${ }^{-1}$ spleens, 23 transcription factors had a binding site in the promoter of genes differentially regulated compared with WT, including downregulation of Nfe2l2, Ppargcla, Ccne1, E2f3, and Nrf1 and upregulation of $I d 2, I d 3, H d a c 1$, and Hdac2 (Supplemental Table 8). Finally, the differentially expressed genes in the Padi2 $2^{-/}$lymph nodes or spleens compared with $\mathrm{Pad}^{-/-}$were enriched with 23 (lymph nodes) and 16 (spleen) transcription factors with a binding site in the promoter of genes differentially regulated (Supplemental Tables 9 and 10). These transcription factors included molecules implicated in type I IFN signaling, antigen presentation, oxidative stress, and neutrophil differentiation. These results indicate that the lack of PAD2 and PAD4 in spleen led to a distinct transcriptional profile regulation compared with lymph nodes and with broader overlap between PAD2 and PAD4 regulation than in lymph nodes.

Alterations in $T$ cell responses in imiquimod-treated $\mathrm{Padi}^{-/-}$and $\mathrm{Padi4}^{-1-}$ mice. To further investigate putative NET-independent roles on the modulation of PAD2 and PAD4 in TLR-7-induced lupus autoimmunity, 
A
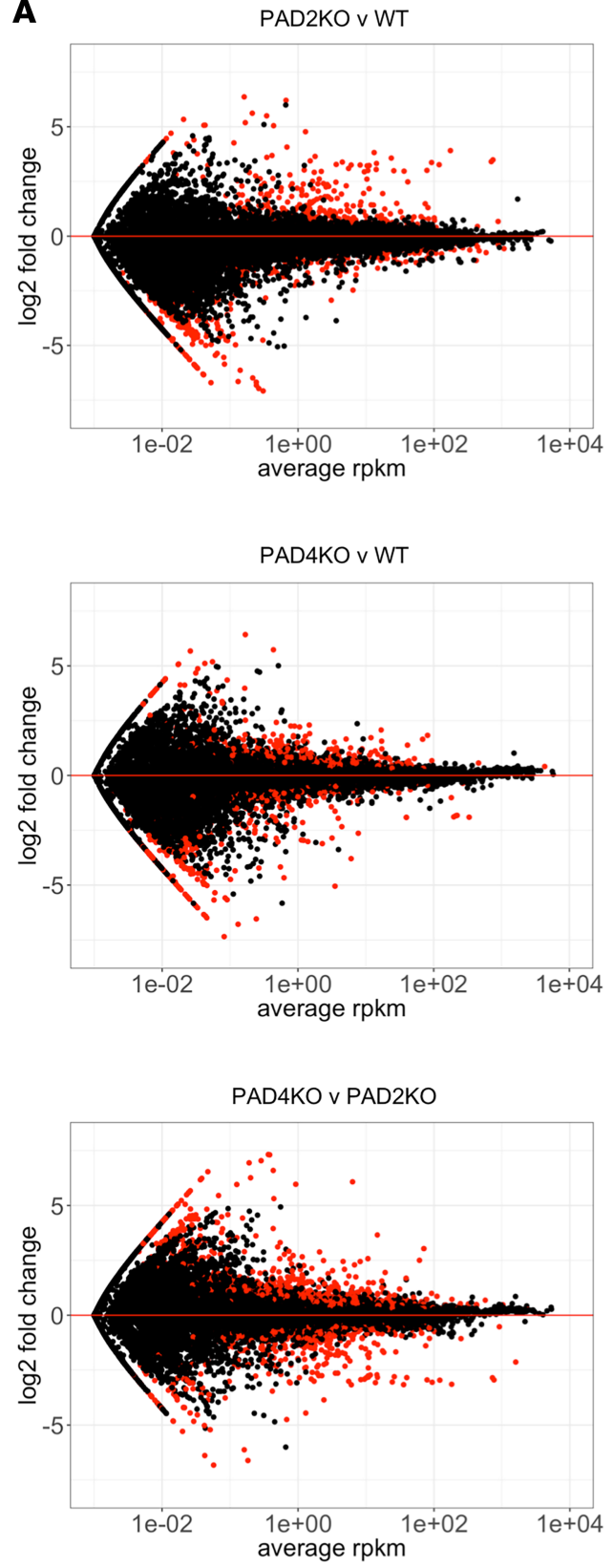

B
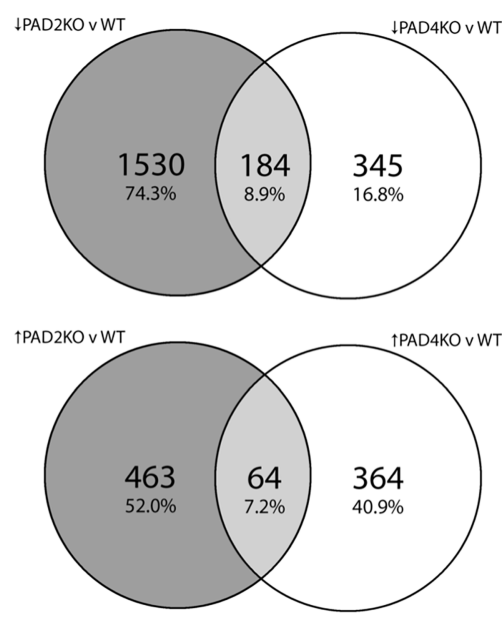

C

GO Biological Pathways: PAD4KO v PAD2KO
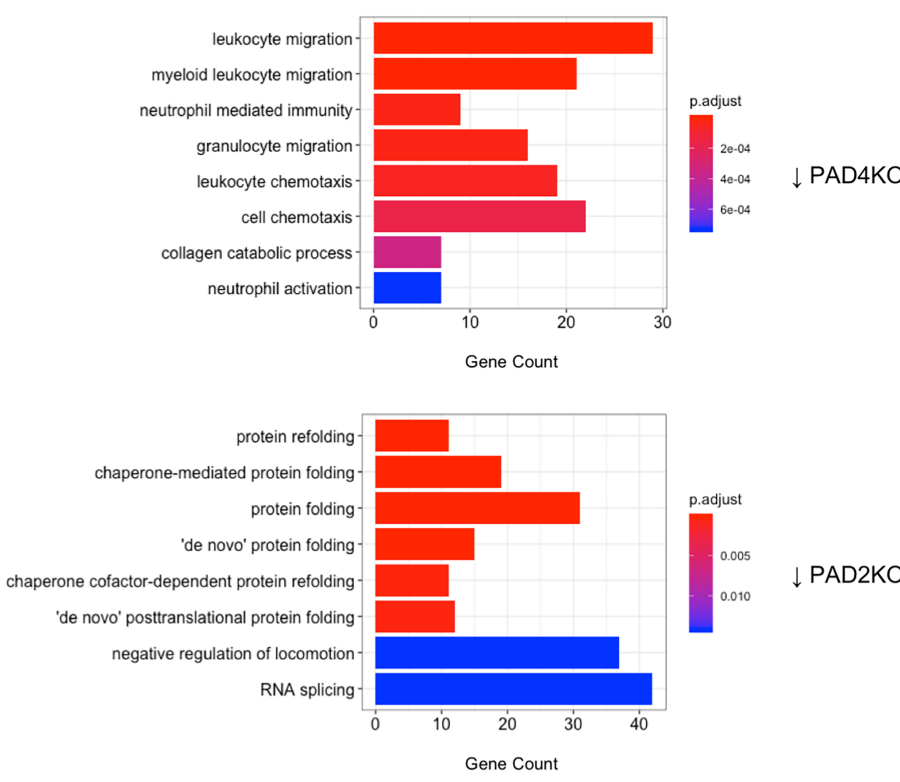

$\downarrow$ PAD2KO

Figure 5. PAD2 and PAD4 differentially regulate gene expression in secondary lymphoid organs. (A) MA plots of gene expression data in lymph nodes following in vivo imiquimod exposure between Padi2 ${ }^{--}$, Padi4 ${ }^{-/}$, and WT FVB (WT) ( $n=4$ mice/group). Differential expression was calculated by ANOVA test on normalized gene counts. Genes with $P<0.05$ are colored in red. (B) Venn diagrams depicting the number of significantly downregulated and upregulated genes between Padiz-/- or Padi4 ${ }^{--}$and WT lymph nodes. (C) Gene Ontology (GO) biological pathway analysis of significant genes between Padi4 ${ }^{-/-}$and Padi2 ${ }^{-/-}$ lymph nodes. Statistical analysis of functional profiles for genes and related pathways was performed using the clusterProfiler package in RStudio.

and given that some of the differentially regulated genes were expressed in lymphocytes (Supplemental Tables 1 and 2), we purified naive and memory $\mathrm{T}$ cells from the various strains exposed to imiquimod. Padi2 mRNA was expressed in both naive and mouse memory T cells at comparable levels, while Padi4 mRNA was detected in memory, but not naive, $\mathrm{T}$ cells (Supplemental Figure 6A). As expected, PAD2 protein was detected in murine $\mathrm{CD}^{+} \mathrm{T}$ cells in $\mathrm{Padi4}^{-/}$and $\mathrm{FVB} \mathrm{CD} 4^{+} \mathrm{T}$ cells but not in $\mathrm{Padi2}^{-{ }^{-}} \mathrm{T}$ cells (Figure 6). PAD4 protein could not be detected in T cells from any of these mouse strains using commercially available antibodies (not shown).

As levels of expression of Padi2 are low in neutrophils and NET formation was not impaired in the absence of this molecule, we explored whether some of the protective effects observed in $\mathrm{Padi}^{-1-}$ were related 
A

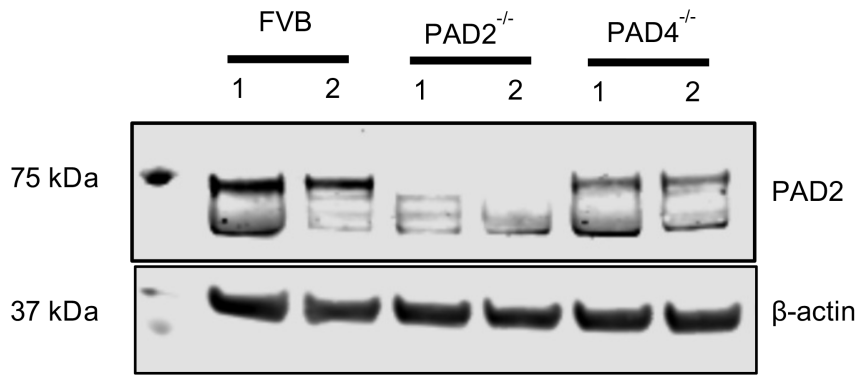

B

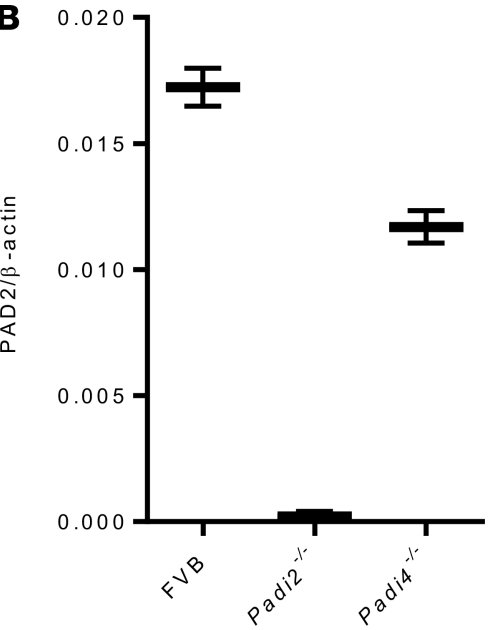

Figure 6. PAD2 expression in murine CD4+ ${ }^{+}$cells. (A and B) Splenocytes isolated from naive FVB, Padi2 ${ }^{-/}$, and Padi4 ${ }^{-/-}$mice were sorted, and PAD2 expression was quantified by Western blot and densitometry. Data are shown as mean \pm SD; 2 mice per group (1 and 2).

to modulation of $\mathrm{T}$ cell responses. Th1 and Th17 responses were downregulated in an ex vivo analysis of imiquimod-treated Padi2 $2^{--}$and Padi4 $4^{--}$mice compared with treated FVB mice (Figure 7, A and B). While no PAD4 protein expression was detected in total $\mathrm{CD}^{+} \mathrm{T}$ cells, Padi4 ${ }^{-1-} \mathrm{T}$ cells displayed decreases in IFN- $\gamma-$ producing $\mathrm{CD} 4^{+} \mathrm{T}$ cells compared with $\mathrm{WT}$ following in vivo imiquimod exposure. These effects were also observed, but were less profound, in Padi2 $2^{--}$mice. These results indicate that both PAD2 and PAD4 modulate in vivo murine $\mathrm{T}$ cell polarization. Given the differences in cellular expression of these PADs, the modulation of $\mathrm{T}$ cell function in the absence of PAD4 could be an indirect effect from other PAD4-expressing innate cells on $\mathrm{T}$ cell function in vivo, consistent with what has been observed in other inflammatory models. To further define whether PAD4's effect on T cell function is an extrinsic phenomenon, in vitro induction of T cell polarization in FVB WT, Padi2 $2^{-/}$, and Padi4 ${ }^{-/}$mice was compared. Consistent with in vivo experiments, Padi $^{-1-} \mathrm{T}$ cells showed impaired abilities to polarize to Th1 and Th17 cells, while this was not the case for Padi4 $^{-1-} \mathrm{T}$ cells (Figure 7C). These results support that the in vivo impairments in mouse $\mathrm{T}$ cell polarization observed in the Padi4 ${ }^{-1-} \mathrm{T}$ cells were secondary to a lack of PAD4 on other immune cell types that modulate $\mathrm{T}$ cell function, rather than on an intrinsic $\mathrm{T}$ cell defect. In contrast, the defects observed in $\mathrm{T}$ cell polarization in Padi2 $2^{--}$murine $\mathrm{T}$ cells appear to be, at least in part, $\mathrm{T}$ cell intrinsic.

$P A D 2$ and PAD4 are expressed in human $C D 4^{+} T$ cells and modulate their function. We next investigated whether PAD expression in human $\mathrm{T}$ cells was similar to their murine counterparts. By flow cytometry, we detected PAD2 expression in CD14 ${ }^{+}$healthy control monocytes, consistent with previous reports (45). While we did not find detectable PAD2 protein in $\mathrm{CD} 15^{+}$human neutrophils, this protein was present in $\mathrm{CD}^{+}$healthy control $\mathrm{T}$ cells (Figure 8A). PAD2 expression was also confirmed in purified SLE CD4 ${ }^{+} \mathrm{T}$ cells by Western blot and immunofluorescence (Figure 8, B and C) with no obvious difference in the levels of this isozyme when comparing control and lupus (Supplemental Figure 6B). Consistent with previous reports, $\mathrm{CD} 15^{+}$neutrophils expressed high levels of PAD4, and in contrast to mouse $\mathrm{T}$ cells, low levels of PAD4 protein were detected in human $\mathrm{CD} 4^{+} \mathrm{T}$ cells (Figure 8D). Furthermore, human memory $\mathrm{CD}^{+} \mathrm{T}$ cells expressed higher levels of both PAD2 and PAD4 protein when compared with naive $\mathrm{CD} 4^{+} \mathrm{T}$ cells (Supplemental Figure 6C). Human and SLE T cells displayed similar levels of PAD4 (Supplemental Figure 6B). Overall, these results suggest different expression of PADs when comparing mouse with human $\mathrm{CD} 4^{+} \mathrm{T}$ cells and activation status of the cell. To test whether PADs are involved in $\mathrm{T}$ cell polarization and cytokine production in human $\mathrm{T}$ cells, isolated $\mathrm{CD} 4^{+} \mathrm{T}$ cells were exposed to the PAD2-specific inhibitor AFM30a (46) and the PAD4-specific inhibitor GSK199 (47). The compounds did not induce CD4+ T cell cytotoxicity at the doses tested, as activation and apoptosis were not affected (Supplemental Figure 7). Human naive $\mathrm{CD}^{+} \mathrm{T}$ cells polarized to $\mathrm{Th} 1$ and $\mathrm{Th} 17$ phenotypes in the presence of these PAD2- and PAD4-specific inhibitors displayed significant reduction in IFN- $\gamma$ production at the mRNA and protein level (Figure 8E), while Th17 polarization was not affected (not shown). These results indicate that, in human $\mathrm{T}$ cells, both PAD2 and PAD4 modulate $\mathrm{T}$ cell function and inhibit Th1 responses. 


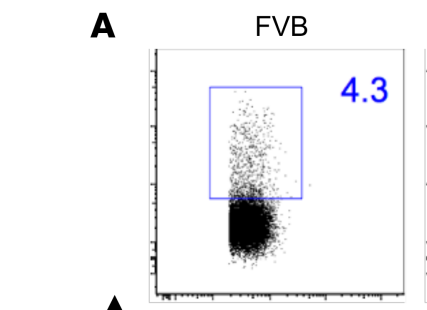

+ IMQ

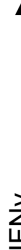
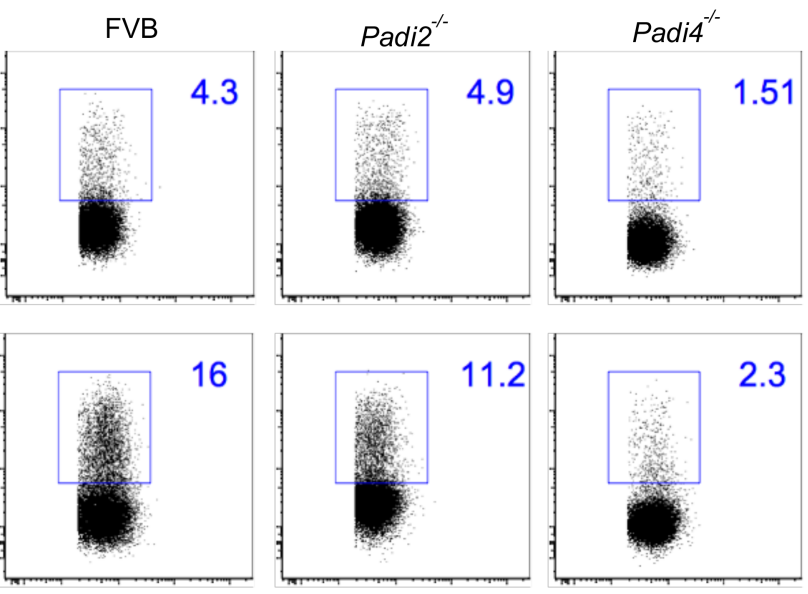

B

FVB
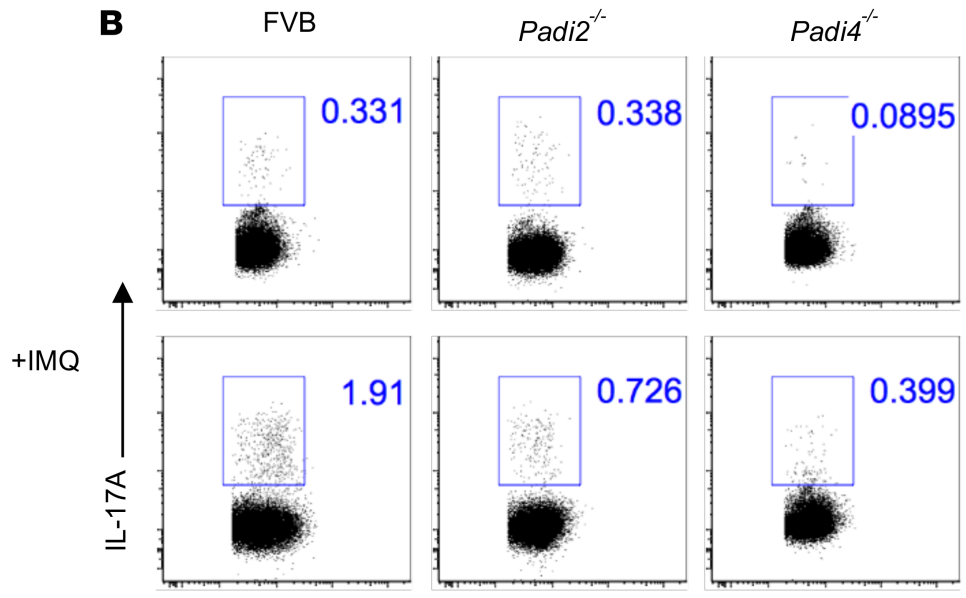
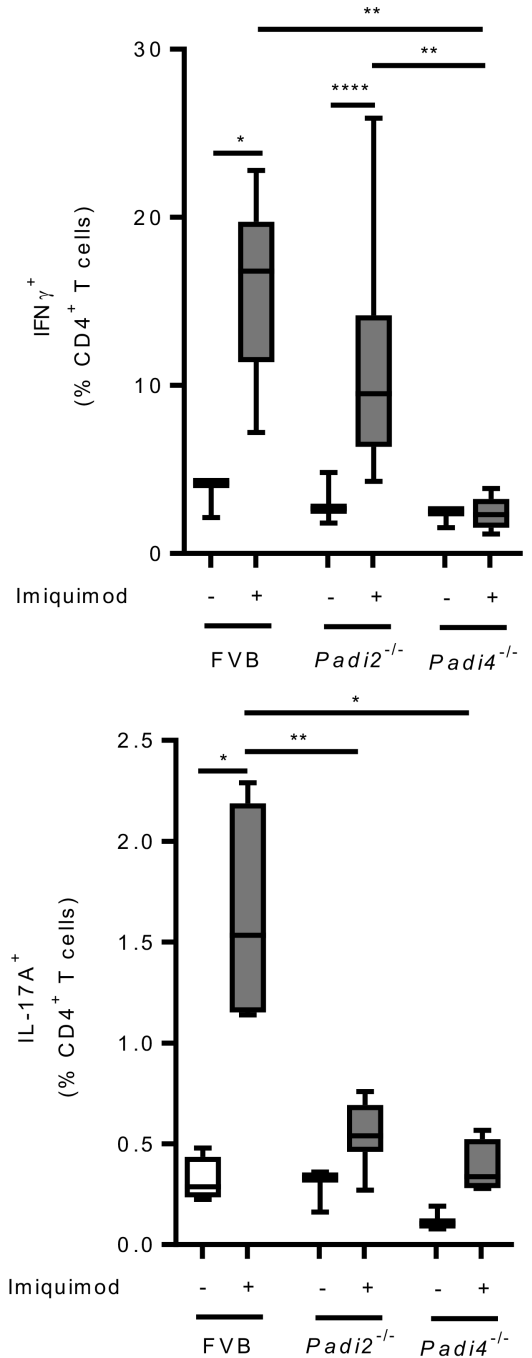

C

Ifng

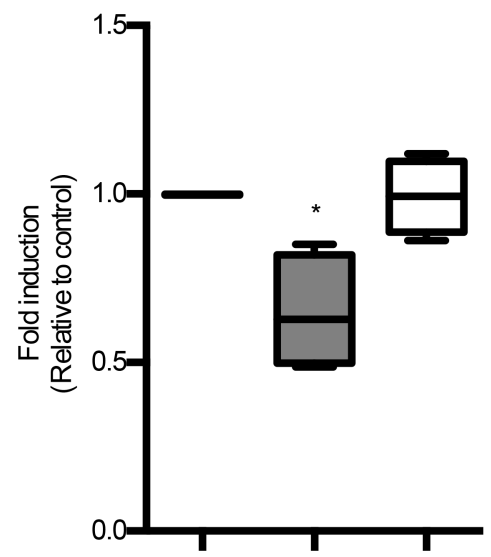

$\| 17 a$

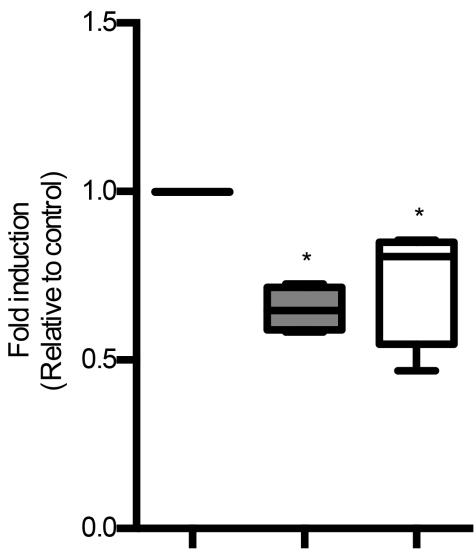

드

Padi2 ${ }^{-1}$

Figure 7. Reduced in vivo Th1 and Th17 responses in imiquimod-treated Padi2 ${ }^{-/-}$and Padi4 ${ }^{-/-}$mice. (A and B) Splenocytes from untreated and imiquimod-treated (IMQ-treated) FVB, Padi2 ${ }^{-/}$, and Padi4 ${ }^{-/-}$mice were stimulated in vitro with PMA and ionomycin, and CD4 ${ }^{+} \mathrm{T}_{\text {cell cytokine production was }}$ measured by flow cytometry to determine Th1 (A) and Th17 (B) responses. Box-and-whisker plots show median, lower and upper quartiles, and minimum and maximum \% values and are representative of 2 independent experiments, each performed in 4-6 mice/group. ${ }^{*} P<0.05,{ }^{* *} P<0.005$, ${ }^{* * * *} P<0.0001$. (C) Splenic naive T cells from FVB, Padi2 $2^{-1-}$, and Padi4 ${ }^{-1-}$ mice were activated by $\alpha$-CD3 and $\alpha$-CD28 in the presence/absence of polarizing cytokines and antibodies. At day 5 , the cells were analyzed for gene expression levels of Ifng in Th1 conditions and I/17a in Th17 conditions; $n=4$. Box-and-whisker plots show median, lower and upper quartiles, minimum and maximum fold induction relative to polarization response in FVB mice. ${ }^{*} P<0.05$, ${ }^{* *} P<0.005$. Statistical analysis was performed by 2-tailed Mann-Whitney $U$ test. 


\section{Discussion}

This study addressed the putative roles that specific PAD enzymes play in the development of TLR-7dependent lupus autoimmunity and its associated organ and vascular damage (Supplemental Table 11). A nonautoimmune-prone mouse background (FVB) was selected to avoid any confounding effects of preexisting immune cell defects. Importantly, circulating levels of different leukocytes in untreated mice were not affected by the lack of either enzyme, independent of their background. Our results demonstrate that, following stimulation with TLR-7 agonists, $\mathrm{Padi4}^{-/}$mice were significantly protected from a variety of features characteristic of lupus autoimmunity, including autoantibody production, type I IFN responses, renal IC deposition, and endothelial dysfunction. In contrast, Padi2 $2^{-/-}$mice displayed a more moderate protection in that they showed lower amounts of autoantibodies and type I IFN responses when compared with WT mice exposed to TLR-7 agonists, but they still exhibited prominent renal IC deposition and endothelial dysfunction. This is somewhat counterintuitive, as PAD2 is more ubiquitously expressed than PAD4 (45) and Padi2 ${ }^{--}$showed more profound changes in gene expression in lymph nodes than Padi4 $^{-1}$ when compared with WT. Moreover, lack of PAD2 or PAD4 did not affect total IgG levels but did selectively decrease levels of various autoantibodies, suggesting that, within the total IgG pool, a subset of autoreactive antibody-producing cells may be preferentially affected by the lack of PADs. Indeed, pathway analysis in lymph nodes suggested that the lack of these molecules modulated canonical pathways of great relevance in B cell ontogeny and activation.

Previously, pan-PAD inhibitors that block both PAD2 and PAD4 effects had been found to be protective in 2 genetically prone murine models of lupus, with similar mitigation of type I IFN responses, renal disease, and endothelial dysfunction $(31,32)$. While these compounds had been found to inhibit NET formation in these animal models, it remained unclear whether PAD4 would solely account for this inhibitory effect, as PAD2 has been reported to also be expressed in neutrophils and have nuclear localization. In the model tested herein, neutrophils deficient in PAD2 were not impaired in their ability to form NETs, while the lack of PAD4 was associated with an inhibitory effect on the synthesis of these traps. In the FVB background, however, the lack of PAD4 still allowed for some neutrophils to form NETs. However, the NETs formed in the absence of PAD4 had significantly reduced Cit-H3 and were found to be significantly less efficient at inducing endothelial dysfunction and type I IFN responses than NETs formed in the presence of PAD4. Whether the decreases in $\mathrm{Cit}-\mathrm{H} 3$ account for all or some of these differences in the properties of the NETs remains to be determined, as PAD4 has the ability to citrullinate several molecules present in neutrophils, including components of the NF-kB pathway, vimentin, and other histones (27). In contrast, NETs formed in the absence of PAD2 appeared to retain their ability to damage the endothelium but were less interferogenic when compared with NETs formed in WT neutrophils. While there are some similarities in the proteins targeted for citrullination by PAD2 and PAD4, some proteins are selectively targeted, and this could account for the differences in the properties of the NETs. A recent report indicates that Cit-H3 promotes dysfunction of the endothelial barrier through reorganization of the actin cytoskeleton (48), and this may explain why mice lacking Padi4 generate NETs devoid of vasculopathic effects. Future studies in mice that genetically lack both PAD2 and PAD4, as well as in vivo use of PAD2- and PAD4-specific inhibitors, will be required to assess whether dual blockade of these pathways promotes synergistic effects in the modulation of autoimmunity and endothelial dysfunction when compared with inhibition of single isozymes, given the effectiveness previously observed with pan-PAD inhibitors in other lupus mouse models $(31,32)$. It will also be important to further assess the putative specific roles of PAD2 and PAD4 in disease phenotype in human SLE.

The mechanism explaining why the absence of PAD2 does not significantly protect mice from IC deposition in the kidney is unclear. Mice lacking either PAD2 or PAD4 had decreases in anti-dsDNA autoantibodies. As we previously proposed for similar observations made using pan-PAD chemical inhibitors, it is possible that decreased NET formation, as occurs in Padi4 $^{-/}$but not in Padi2 ${ }^{--}$, lowers the amounts of generated autoantigens required for IC formation (31). This, combined with lower anti-dsDNA synthesis in these mice, may lead to a decrease in renal IC deposits. In contrast, given that $\mathrm{Padi2}^{-1-}$ are less protected from enhanced NET formation, they may generate sufficient amounts of autoantigen to increase IC deposition, even if some types of autoantibodies decrease.

Two recent studies in other mouse models of lupus recently challenged the concept that PAD4 is an important target in SLE $(34,35)$. However, some of the models used, like the MRL/lpr model, are primarily characterized by the expansion of aberrant autoreactive $\mathrm{T}$ cells due to a lack of functional Fas (49). Given that we did not find that PAD2 and PAD4 inhibitors affected T cell survival, it is possible 
A

PAD2

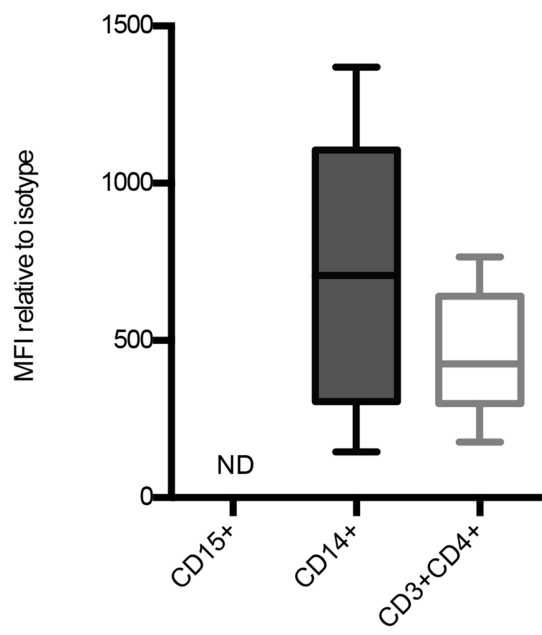

B

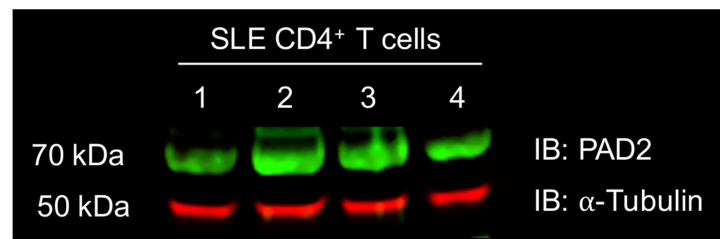

C

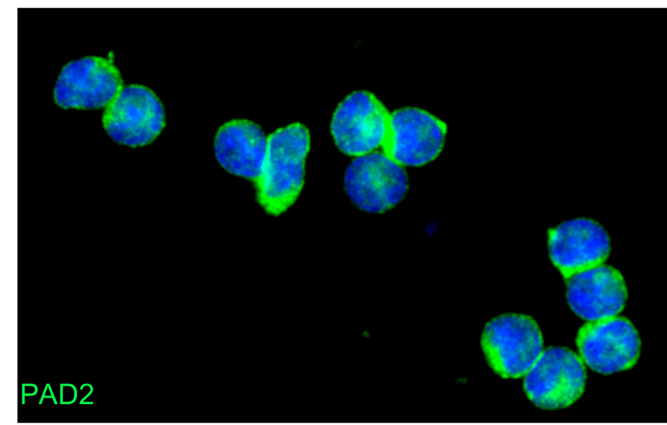

D

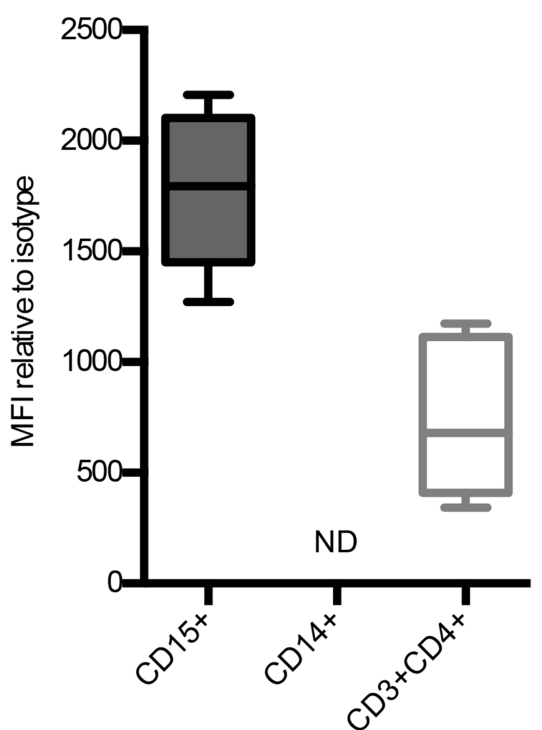

E

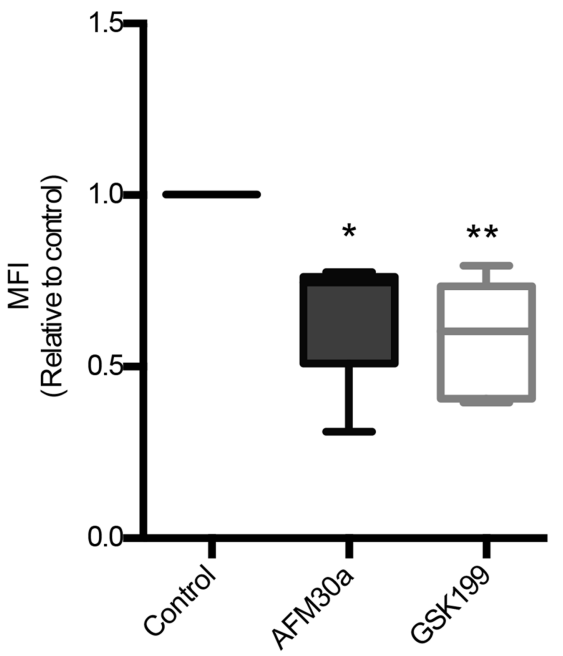

Ifing

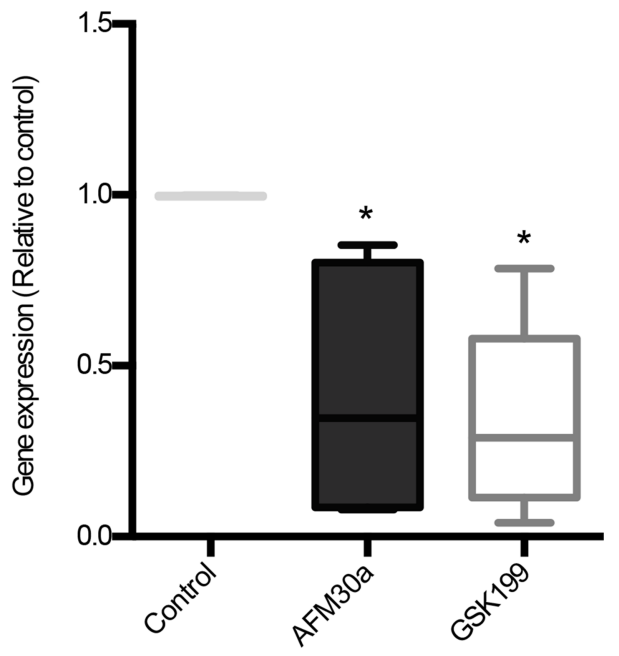

Figure 8. PAD2 and PAD4 are expressed on human T cells and modulate Th1 responses. (A) PAD2 protein expression levels (by geometric mean fluorescent intensity, MFI, relative to isotype control) in healthy control human neutrophils (CD15+), monocytes (CD14+), and T cells (CD3+CD4+). Box-and-whisker plots show median, lower and upper quartiles, and minimum and maximum MFI values of 5 independent experiments. (B and C) PAD2 protein expression quantified in SLE CD4 ${ }^{+}$T cells by Western blot (B) and immunofluorescence (C). In B, each lane represents an individual SLE subject ( $\left.n=4\right)$. C is a representative microphotograph of lupus CD4+ T cells $(n=2)$; magnification is 40x. (D) PAD4 protein expression levels assessed by geometric MFI relative to isotype control in healthy control human neutrophils $\left(C D 15^{+}\right)$, monocytes $\left(C D 14^{+}\right)$, and T cells $\left(C D 3^{+} C D 4^{+}\right)$. Box-and-whisker plots show median, lower and upper quartiles, and minimum and maximum MFI values of 5 independent experiments. (E) Sorted healthy donor naive CD4+ T cells were activated with $\alpha$-CD3 and soluble $\alpha$-CD28 and polarized for 5 days in the presence or absence of the PAD2 inhibitor AFM30a or the PAD4 inhibitor GSK199. Left graph shows box-and-whisker plots displaying median, lower and upper quartiles, and minimum and maximum MFI values of IFN- $\gamma$ in Th1 conditions, and right graph displays box-and-whisker plots with median, lower and upper quartiles, and minimum and maximum mRNA fold induction of IFNG in Th1 conditions relative to untreated control; $n=5$ healthy controls. Statistical analysis was performed by 2-way ANOVA with Dunnett's multiple comparisons test.

that the lack of PAD4 is not sufficient to abrogate disease in a model primarily driven by aberrant autoreactive $\mathrm{T}$ cells. Indeed, previous studies suggest that dual inhibition with a pan-PAD inhibitor, which may target $\mathrm{T}$ cells more effectively given our observations in the current study, mitigates renal disease, skin involvement, and immune dysregulation in MRL/lpr (32). Given that PAD2 is the primary PAD expressed in murine $\mathrm{T}$ cells, it is not surprising that single deletion of PAD4 would not alter the natural history of lupus in this model. In the same manuscript, the authors also reported that the anti-glomerular 
basement membrane (anti-GBM) nephritis model was not protected when a pan-PAD inhibitor was administered (34). It is important to note that the anti-GBM model is more reminiscent of a rapidly progressive glomerulonephritis than of classic lupus nephritis and also lacks many of the features of SLE such as anti-dsDNA autoantibodies or type I IFN responses (50). In the anti-GBM model, neutrophils are abundant in the kidney (51), while they are fairly scant in renal tissue of genetically driven murine lupus models and in human $\operatorname{SLE}(21,31,52)$. It is also important to note that - when there is very significant influx of neutrophils into a tissue and, therefore, high neutrophil density - NETs undergo a phenomenon of aggregation (53) that can lead to an antiinflammatory effect due to the cleavage of inflammatory molecules by neutrophil proteases. NET aggregation is a common finding of conditions such as gout (53) or the peritoneum of pristane-induced lupus (35), but it is not characteristic of other murine lupus models or human $\operatorname{SLE}(21,31)$. Therefore, the proinflammatory versus antiinflammatory effects of NETs are very likely context dependent and may explain, at least in part, both the discrepant effects observed in the various mouse models studied and the heterogeneity of human SLE.

An additional aspect of this study is the characterization of the role of PAD2 and PAD4 in T cell responses in TLR-7-dependent autoimmunity and in human T cells. While both Padi2 and Padi4 mRNA were detected in memory murine T cells, PAD4 protein was not. This is consistent with results from a previous study on the role of PAD4 in the glucose-6-phosphate isomerase-induced (GPI-induced_arthritis model where impairments in Th17 cell differentiation observed in Padi4 ${ }^{-1}$ were also due to an extrinsic factor during GPI immunization, rather than to intrinsic defects in Padi4 ${ }^{-/-}$murine $\mathrm{CD} 4{ }^{+} \mathrm{T}$ cells (54). In contrast, the effects of PAD2 on T cell polarization appear to be, at least in part, intrinsic, given that $\mathrm{T}$ cell polarization in the context of PAD2 deficiency was inhibited in vivo and in vitro. Importantly, the effect of PAD4 on human T cells differs from mice. Both PAD4 and PAD2 protein were found to be expressed in human T cells, with memory $\mathrm{T}$ cells expressing higher levels than naive T cells. Importantly, both PAD2- and PAD4-specific inhibitors were able to decrease Th1 polarization in vitro, suggesting that these compounds may target Th1-driven immunity. This is a relevant finding in the context of several autoimmune diseases that are driven by both abnormalities in the innate and adaptive immune arms, such as SLE. Th1 cells have been described to play important roles in SLE and in lupus-associated organ damage such as lupus nephritis $(55,56)$. Therefore, targeting Th1 cells by PAD inhibition may add a beneficial protective effect in SLE. It will be important in future studies to understand the molecular mechanisms by which PADs impact Th1 polarization. Of note, a recent study described PAD2 expression in human neutrophils (28). In our studies, we were not able to detect PAD2 protein in human neutrophils. This could be explained by the recent observation that PAD2 is actively secreted by neutrophils, supporting why PAD2 was not detected by intracellular flow (28).

Detailed RNA-Seq analysis revealed distinct transcriptional modulation in response to in vivo TLR-7 stimulation when comparing lymph nodes and spleens in mice lacking PAD2 and PAD4. The differences observed between the pathways modulated in spleens and lymph nodes by these PADs are likely the reflection of the cells that are most predominant in these organs following TLR-7 stimulation. Future studies should further address how these PADs modulate gene expression and cellular function, including metabolism, in specific cell subsets purified from lymphoid organs. The pathway analysis does support that PAD4 influences autoimmunity, at least in part, through significant modulation of neutrophil responses, while PAD2 may be relevant to inflammation through regulation of other myeloid cell responses and changes in the adaptive immune compartment. Future studies should systematically investigate the mechanisms leading to differential regulation of gene expression mediated by different PADs in immune cells using purified cell subsets and epigenetic analyses to further assess differential contributions of each PAD to innate and adaptive immune responses.

In summary, we have identified differential regulation and modulation of autoimmune responses, gene expression in secondary lymphoid organs, and endothelial dysfunction by the isozymes PAD2 and PAD4 in TLR-7-dependent autoimmunity and in potentially pathogenic T cell responses. Given that both isozymes appear to play differential roles in the modulation of immune responses, the use of chemical inhibitors that target both PAD2 and PAD4 should be further examined for the potential to provide complimentary therapeutic effects in the treatment of autoimmune conditions characterized by aberrant innate and adaptive immune responses such as SLE. 


\section{Methods}

Animals. WT FVB mice were obtained from the Jackson Laboratory and housed at the NIAMS animal facility throughout the experimental period. KO mice were generated in the FVB genetic background. The gene-trapped Padi2-null mice were obtained from the Texas Institute for Genomic Medicine. Padi2null mice were generated by partial replacement of the first coding exon with the IRES/bGoe/PolyA cassette via homologous recombination. The deleted genomic sequence is as follows: 5' - GCAGCCGCCTATACGGGAAAATATGCTGCGGGAACGGACCGTGCGGCTGCAGTATGGGAGCCGCGTGGAGGCGGTGTACGTGCTGGGCACGCAGCTCTGGACCGATGTCTACAGGTGAGCCGAGAGAGAGTTACCCTCTCACCTACCCTTCACAAATCTTGAG - 3'. To generate the Padi4-null mice, the genomic sequence of Padi4 from the ATG start site to approximately $100 \mathrm{bp}$ downstream of the TGA stop codon was replaced in frame (with respect to the Padi4 initiation codon) by the coding sequence of LacZ and a Lox-flanked neomycin gene driven by the PGK-EM7 promoter. The deleted genomic sequence is as follows: 5' - GGCTAAGGGCATAGCCTGTGCTCTCCCCTCACAGGCTTCCGAGTGCCCCACTCTTCTGCTGTTGCAGGCTTCACTGTGGACACCCATGGGTGGGTGGGTGGGGGTCCTGGGTGGCCCAGGGTAGAGGAGGCTGTGCAGCCACTGGGGCACCTGAGTGGAGTGGGGCTGGGCCTCCGGGGCAGGGCAGGTGCTGACTCATGGGCTCGCCTGGCACTATAAAGGAACCAGCCCAGGGGCTTCCTGCTGCCTGCTGACCGAGCGAGCAAGGATG// GCCCAAGGCGCGGTGATCCACGTGGCCCCCGAGCAGCCCACTCACGCCGTGTGTGTGGTGGGCACAGCGACCCCGCTGGATGTCCGCGGGTGAGAGGGGCAGAGATCCTGGGCCTGTGGGGGCAGTTTGGGGCAGGCAGGAGCTGGGCGCTGCTG - 3'. Genotyping confirmed the genetic background of these strains.

Human subjects. Patients fulfilled the American College of Rheumatology revised diagnostic criteria for SLE (57), and more than 90\% were females. Disease activity was assessed by the SLE Disease Activity Index (SLEDAI) score (58). The mean SLEDAI was 4 (range 1-18), indicating mild disease activity. Peripheral blood was drawn by venipuncture in EDTA-containing tubes. Human PBMCs were isolated by Ficoll-Paque Plus (GE Healthcare) density gradient.

Generation of imiquimod-induced lupus model. Female 6- to 8-week-old mice were treated topically with the TLR-7 agonist imiquimod, which induces systemic autoimmunity with lupus-like characteristics in various non-autoimmune-prone mice strains $(37,41)$. Imiquimod cream 5\% (E Fougera \& Co) was applied to both ear folds of WT, Padi2 ${ }^{-/}$, and Padi4 ${ }^{--}$FVB mice 3 times a week. During the third week of treatment, mice were exposed to a subacute dose of $300 \mathrm{~mJ} / \mathrm{cm}^{2}$ UVB-narrowband radiation on shaved dorsal skin for 5 consecutive days. Disease severity was analyzed after 6 weeks from the onset of treatment.

Quantification of blood cell counts and autoantibodies. Whole blood was collected at euthanasia by cardiac puncture, and serum was purified. Complete blood counts were measured at the Department of Laboratory Medicine (NIH). Commercially available ELISA kits were used to quantify serum anti-dsDNA and total IgG levels (Alpha Diagnostic International), per manufacturer's instructions. Serum anti-RNP/Sm and anti-histone autoantibodies were also determined by ELISA, as described (45). Nunc MaxiSorp ELISA plates were precoated with recombinant $\mathrm{RNP} / \mathrm{Sm}$ (RNP/Sm; $20 \mathrm{mg} / \mathrm{ml}$, ImmunoVision) or calf thymus histone (20 mg/ $\mathrm{ml}$, MilliporeSigma) in PBS at $4^{\circ} \mathrm{C}$ overnight. Plates were blocked with $3 \% \mathrm{FCS}$ for 1 hour at $37^{\circ} \mathrm{C}$, washed, and incubated with 1:100 dilutions of mouse sera for 1 hour at $37^{\circ} \mathrm{C}$. Plates were washed, and specific antibodies were detected with a $1 / 1000$ dilution of alkaline phosphatase-conjugated goat anti-mouse IgG (103605; Southern Biotech) for 1 hour at $37^{\circ} \mathrm{C}$ and developed with a phosphatase substrate for 30 minutes at $37^{\circ} \mathrm{C}$.

Renal disease assessment. Urine samples were collected at euthanasia, and albumin/creatinine ratio was quantified using the Albuwell and Creatinine Companion kits (Exocell). Kidneys were perfused with saline and preserved in OCT compound. For glomerular IC analysis, frozen sections were analyzed for C3 and IgG deposition. Frozen tissue samples were stained with FITC-conjugated anti-C3 (ICL) and Alexa Fluor 594-conjugated anti-mouse IgG (Thermo Fisher Scientific) for 1 hour at $4^{\circ} \mathrm{C}$. DNA was visualized using Hoechst (Thermo Fisher Scientific). ICs were scored in a blinded fashion as described (59). A scale of 0-3 was assigned for both C3 and IgG deposition based on intensity of signal, as follows: 0, normal (no staining); 1, mild; 2, moderate; and 3 , severe. A minimum of 10 glomeruli were scored per mouse, and the individual average scores were reported. Quantification of renal inflammation and damage was performed by a veterinary pathologist as described (59).

Neutrophil isolation and NET formation assay. BM-derived neutrophils were isolated from untreated FVB, Padi2 ${ }^{-/}$, or Padi4 ${ }^{--}$mice by negative selection using EasySep Mouse Neutrophil Enrichment Kit (Stemcell Technologies). Neutrophils $\left(1 \times 10^{5}\right)$ were allowed to adhere to coverslips coated with poly-L-lysine (MilliporeSigma) 
and were then treated with the calcium ionophore A23187 (50 $\mu \mathrm{M}$; Thermo Fisher Scientific) in serum-free RPMI 1640 (Thermo Fisher Scientific) for 2 hours at $37^{\circ} \mathrm{C}$. Neutrophils were then fixed in $4 \%$ paraformaldehyde in PBS (Thermo Fisher Scientific) at $4^{\circ} \mathrm{C}$ overnight. Nonspecific binding was blocked with $0.02 \%$ porcine skin gelatin (MilliporeSigma) in PBS for 30 minutes. Cells were incubated with anti-MPO (1:1000; Agilent Technologies) or anti-Histone H3 (citrulline R2 + R8 + R17; Cit-H3; 1:250; Abcam) diluted in blocking solution for 1 hour at $37^{\circ} \mathrm{C}$ in a humidified chamber. After 3 washes, coverslips were incubated with Alexa Fluor $488-$ or Alexa Fluor 555-conjugated secondary antibodies (1:500; Thermo Fisher Scientific) diluted in blocking solution for 30 minutes at $37^{\circ} \mathrm{C}$ in a humidified chamber. Hoechst-stained (1:1000) coverslips were mounted onto glass slides with ProLong Gold Antifade Mountant (Thermo Fisher Scientific) and visualized after curing overnight. The frequency of NET-forming neutrophils was quantified by determining the number of neutrophils extruding DNA and containing at least 1 protein associated with NETs (i.e., MPO and/or CitH3), in relation to the total number of neutrophils analyzed. A minimum of 3 images were quantified per sample.

NET isolation and protein analysis. Neutrophils were isolated from untreated FVB, Padi2 ${ }^{-1-}$, or Padi4 ${ }^{-1-}$ mice and stimulated with imiquimod (R837; $100 \mu \mathrm{g} / \mathrm{ml}$; InvivoGen) in serum-free RPMI 1640 for 6 hours at $37^{\circ} \mathrm{C}$. NETs were isolated as previously described (47) using $10 \mathrm{U} / \mathrm{ml}$ of micrococcal nuclease (Thermo Fisher Scientific) for 15 minutes at $37^{\circ} \mathrm{C}$ (48). NETs were centrifuged to remove cellular debris and the supernatants stored at $-80^{\circ} \mathrm{C}$ until used. NETs were separated using a NuPAGE 4-12\% Bis-Tris Protein Gel (Thermo Fisher Scientific) and transferred onto a nitrocellulose membrane (Bio-Rad). The membrane was blocked with 10\% BSA for 30 minutes at room temperature (RT) and was then incubated with a mouse monoclonal antibody targeting total Histone $\mathrm{H} 3$ (Abcam) and a rabbit polyclonal antibody specific for Cit-H3 (Abcam) overnight at $4^{\circ} \mathrm{C}$. The membrane was washed 3 times with PBS-Tween $(0.1 \%)$ and incubated with secondary antibodies coupled to IRDye 800CW and IRDye 680RD (Thermo Fisher Scientific) accordingly. After 3 additional washes, the membrane was developed using a Li-COR Odyssey Clx scanner (Li-COR Biosciences).

Endothelium-dependent vasorelaxation assays. Studies were performed as described by our group (59). After euthanasia, endothelium-intact aortic rings $(\sim 2 \mathrm{~mm}$ ) were excised and mounted in a myograph system (Danish Myo Technology A/S) containing physiological salt solution (PSS) with aeration $\left(95 \% \mathrm{O}_{2} / 5 \% \mathrm{CO}_{2}\right.$ ). Aortic rings were subsequently set at $700 \mathrm{mg}$ passive tension and equilibrated for 1 hour with buffer changes every 20 minutes. Contraction was achieved with PSS containing $100 \mathrm{mM}$ potassium chloride (KPSS) prior to collecting contraction/relaxation measurements. Phenylephrine-induced (PE-induced) contraction was allowed to reach a stable plateau. Vasorelaxation was assessed by addition of Ach $\left(1 \times 10^{-9} \mathrm{M}\right.$ to $\left.1 \times 10^{-5} \mathrm{M}\right)$. To assess endothelial dysfunction induced by NETs, FVB aortas were incubated with imiquimod-generated NETs for 15 minutes prior to the vasorelaxation assay. Similar amounts of NETs were included in the assay, as shown by total histone $\mathrm{H} 3$ analysis (Figure 4C). Results were reported as percentage of PE contraction.

Extracellular flux assay. A total of $3 \times 10^{5} \mathrm{BM}$ mouse neutrophils were seeded onto poly-L-lysine-coated Seahorse XF96 microplates in glucose-free XF Base Medium (Agilent Technologies) supplemented with 4 mM GlutaMAX (Thermo Fisher Scientific) and $1 \mathrm{mM}$ sodium pyruvate (Thermo Fisher Scientific). The XF96 Analyzer (Agilent Technologies) was used to assay the cells. Glucose (MilliporeSigma) was injected in Port A at a final concentration of $25 \mathrm{mM}$, and 2-Deoxy-D-glucose (2-DG; MilliporeSigma) was injected in Port $\mathrm{C}$ at a final concentration of $100 \mathrm{mM}$. Data were analyzed with the Wave Desktop software. The extracellular acidification rate (ECAR) was used as an indicator of glycolysis. Glycolysis was defined as the ECAR at baseline subtracted from the ECAR after glucose injection.

Splenic proinflammatory cell analysis. Mouse splenocytes were isolated from untreated and imiquimod-treated FVB, Padi2 ${ }^{-/}$, or Padi4 ${ }^{--}$mice and stimulated in vitro with phorbol 12-myristate 13-acetate (PMA; $50 \mathrm{ng} / \mathrm{ml}$; MilliporeSigma) and ionomycin (750 $\mu \mathrm{g} / \mathrm{ml}$; MilliporeSigma) for 3 hours. Brefeldin A (BFA; BioLegend) was added for an additional hour, and LIVE/DEAD Fixable Blue Dead Cell Stain Kit (Thermo Fisher Scientific) was used to label dead cells prior to incubating with FcR Blocking Reagent (Miltenyi Biotec). Splenocytes were stained with anti-CD3-PE-Cy5 (145-2C11) and anti-CD4-Brilliant Violet 605 (RM4-5). The Fixation/Permeabilization Solution Kit (BD Biosciences) was used before intracellular staining with anti-IL-17A (TC11-18H10.1) and anti-IFN- $\gamma$ (XMG1.2). All antibodies were from BioLegend. Data were collected with the BD LSRFortessa (BD Biosciences) and analyzed with FlowJo.

Mouse $T$ cell activation and polarization assays. Naive $\mathrm{CD} 4{ }^{+} \mathrm{CD} 62 \mathrm{~L}^{+} \mathrm{CD} 44^{-} \mathrm{T}$ cells were isolated from 10-week-old FVB, Padi2-/- or Padi4 ${ }^{-1}$ spleens by sorting with FACSAria IIIu. Cells were stimulated for 4 hours with PMA $(50 \mathrm{ng} / \mathrm{ml})$ and ionomycin $(750 \mu \mathrm{g} / \mathrm{ml})$, with the addition of BFA, and processed with the Fixation/Permeabilization Solution Kit after surface staining. Cells were stained with anti-CD4-BV421 
(GK1.5), anti-CD62L-PE (MEL-14), anti-CD44-APC-Cy-7 (IM7), anti-IFN- $\gamma$-FITC (XMG1.2), and anti-IL-17A-APC (TC11-18H10.1). All staining antibodies were purchased from BioLegend. Data were collected with the BD LSRFortessa and analyzed with FlowJo.

Mouse $\mathrm{T}$ cell in vitro polarization was performed as described (60). CD4 ${ }^{+} \mathrm{CD} 62 \mathrm{~L}^{+} \mathrm{CD} 44^{-}$naive splenic T cells from 10-week-old FVB, Padi2 ${ }^{-1-}$, or $\mathrm{Padi4}^{--}$mice were sorted using FACSAria IIIu and activated by plate-bound $10 \mu \mathrm{g} / \mathrm{ml} \alpha-\mathrm{CD} 3$ and $10 \mu \mathrm{g} / \mathrm{ml} \alpha-C D 28$ (BioLegend) in T cell culture medium (RPMI1640 with 10\% FBS [Gemini Bio], 2 mM glutamine [Thermo Fisher Scientific], penicillin [100 IU/ml] [Thermo Fisher Scientific], streptomycin [0.1 mg/ml] [Thermo Fisher Scientific], $10 \mathrm{mM}$ HEPES [MilliporeSigma], $1 \mathrm{mM}$ sodium pyruvate [Thermo Fisher Scientific], and $50 \mu \mathrm{M} \beta$-mercaptoethanol [Bio-Rad]) for 3 days in the presence/absence of various cytokines and antibodies as indicated. At day 3, cells were removed and rested with IL-2 and fresh cytokines for 2 more days. Conditions were as follows: (Th0) anti-IFN- $\gamma(10 \mu \mathrm{g} / \mathrm{ml}$; catalog 506502, clone B27) and anti-IL-4 (10 $\mu \mathrm{g} / \mathrm{ml}$; catalog 500707, clone 8D4-8); (day 3) IL-2 (100 U/ml; catalog 589108), anti-IFN- $\gamma(10 \mu \mathrm{g} / \mathrm{ml})$, and anti-IL-4 (10 $\mu \mathrm{g} / \mathrm{ml})$; (Th1) IL-12 (10 ng/ml) and anti-IL-4 (10 $\mu \mathrm{g} /$ $\mathrm{ml})$; (day3) IL-2 (100 U/ml), IL-12 (10 ng/ml), and anti-IL-4 $(10 \mu \mathrm{g} / \mathrm{ml})$; and (Th17) IL-6 (20 ng/ml; catalog 573008), TGF- $\beta$ (3 ng/ml; R\&D systems, catalog 240-B-010), IL-23 (50 ng/ml; catalog 574106), anti-IFN- $\gamma$ $(10 \mu \mathrm{g} / \mathrm{ml})$, and anti-IL-4 $(10 \mu \mathrm{g} / \mathrm{ml})$. All antibodies were purchased from BioLegend. For intracellular cytokine staining, cells were restimulated for 4 hours with PMA $(50 \mathrm{ng} / \mathrm{ml})$ and ionomycin $(750 \mu \mathrm{g} / \mathrm{ml})$, with the addition of monensin (BD GolgiStop). Cells were stained for CD4, CD62L, CD44, IFN- $\gamma$, and IL-17A.

RNA-Seq. Inguinal lymph nodes and spleens were collected from imiquimod-treated mice at euthanasia and frozen at $-80^{\circ} \mathrm{C}$ in Tri-Reagent (MilliporeSigma). RNA was isolated with Direct-zol RNA MiniPrep (Genesee El Cajon) and cDNA libraries prepared with poly (A) tail enrichment using NEBNext Ultra II RNA Library Prep Kit following manufacturer's instructions (New England Biolabs; E7770S and E7490S). The fragmentation of the libraries was assessed with Agilent Technologies' D1000 ScreenTape and concentration quantified with picogreen (Agilent Technologies). RNA-Seq data were generated with Illumina HiSeq3000 for 50 cycles (single-end reads). Raw sequencing data were processed with bcl2fastq/2.17.1 to generate FastQ files. Adapter sequences were removed using trimgalore/0.4.5. Single-end reads of 50 bases were mapped to the mouse genome build mm10 using TopHat2.1.1 (61). Gene expression values (reads per kilobase exon per million mapped reads; RPKM) were calculated with Partek Genomics Suite 6.6, which was also used for 1-way ANOVA analyses. Gene expression data were subsequently analyzed in R Studio with a $P$ value cutoff of 0.05 . MA plots were generated using the ggplot2 package. Pathway analyses were performed using the clusterProfiler package and gene ontology (GO) database (62). Transcription factor analyses were generated with IPA (Ingenuity Pathway Analysis, QIAGEN, V01-10) software. The data has been deposited into GEO (GSE121751).

Real-time PCR. Splenocytes were isolated from untreated and imiquimod-treated FVB, Padi2 ${ }^{-1-}$, or Padi4-1mice. Cell pellets were collected in TRI Reagent (MilliporeSigma), and RNA was isolated with the Direct-zol RNA MiniPrep Kit (Zymo Research). RNA was transcribed into cDNA using iScript Reverse Transcription Supermix (Bio-Rad). CXF96 Touch Real-Time PCR Detection System was used to amplify the following transcripts: Ifna (forward, 5' - AAG GAC AGG CAG GAC TTT GGA TTC - 3'; reverse, 5' - GAT CTC GCA GCA CAG GGA TGG - 3'), Ifnb (forward, 5' - AAG AGT TAC ACT GCC TTT GCC ATC - 3'; reverse, 5' CAC TGT CTG CTG GTG GAG TTC ATC - 3'), Irf7 (forward, 5' - TCC AAG TCT CCG GCT AAG T - 3'; reverse, 5' - TGC TGT TTG GAG ACT GGC TAT - 3'), and $M x 1$ (forward, 5' - GAT CCG ACT TCA CTT CCA GAT GG; reverse, 5' - CAT CTC AGT GGT AGT CAA CCC - 3'). Values were normalized to Gapdh expression, and fold change was shown as expression in imiquimod-treated mice relative to expression in untreated mice. To test the effects of NETs of type I IFN-related gene expression, FVB splenocytes were isolated and cultured for 24 hours with and without imiquimod-generated NETs from FVB, Padi2 ${ }^{--}$, or Padi4 ${ }^{-1}$ neutrophils (5\%). Imiquimod at a concentration corresponding to that present in the NET preparations was used as the vehicle control $(5 \mu \mathrm{g} / \mathrm{ml})$. Cells were collected and processed as described above. The following primer sets were used: Isg15 (forward, 5' - CAG AAG CAG ACT CCT TAA TTC - 3'; reverse, 5' - AGA CCT CAT ATA TGT TGC TGT G - 3'), Tbk1 (forward, 5' - TTC TGG AAG TCC AGA CGC ATTG - 3'; reverse, 5' - ACT GGT GAT CTC TAT GCT GTC A - 3'), and Gapdh (forward, 5' - ACC ACA GTC CAT GCC ATC AC - 3'; reverse, 5' - TCC ACC ACC CTG TTG CTG TA - 3'). Samples were normalized to Gapdh, and fold change was expressed as treated (vehicle or NETs) compared with untreated cells.

To measure the expression of PADs in murine T cells, 9-week-old female WT, Padi2-/-, and Padi4 ${ }^{-1}$ FVB mice were treated topically with imiquimod for 2 weeks. Splenic CD4 ${ }^{+} \mathrm{CD} 62 \mathrm{~L}^{+} \mathrm{CD} 44^{-}$naive $\mathrm{T}$ cells and $\mathrm{CD} 4{ }^{+} \mathrm{CD} 62 \mathrm{~L}^{-} \mathrm{CD} 44^{+}$memory $\mathrm{T}$ cells were sorted using FACSAria IIIu, and expression of the 2 genes 
analyzed with Padi2- or Padi4-specific TaqMan Gene Expression Assays (Padi2 [Mm01341648_m1] and Padi4 [Mm01341658_m1]; Applied Biosystems) and normalized to 18s ribosomal RNA (Mm03928990_g1). Gene expression data were confirmed by protein analysis. $\mathrm{CD} 4^{+} \mathrm{T}$ cells were isolated from mice, and PAD2 was detected by immunofluorescence and Western blotting (Proteintech Group). For human T cells, healthy donor $\mathrm{CD}^{+} \mathrm{T}$ cells were stained with anti-CD4-BV421 (catalog 317434, clone OKT4, BioLegend), -CD45RA-PerCP-Cy5.5 (catalog 304122, clone HI100, BioLegend), and-CD45RO-APC-H7 (catalog 561137, clone UCHL-1, BD Biosciences) before sorting with the FACSAria IIIu. Naive T cells $\left(\mathrm{CD} 4^{+} \mathrm{C}\right.$ -

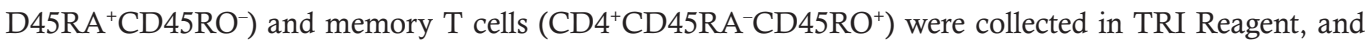
RNA was isolated and transcribed as described above. PADI2 (Hs01042505_m1) and PADI4 (Hs01057483_ m1) were measured and normalized to $18 S$ ribosomal RNA (Hs03003631_g1). For T cell polarization studies, T cells were collected and processed as described above. TaqMan Gene Expression Assays used were as follows; (mouse) 18S, Mm03928990_g1; ifng, Mm01168134_m1; and Il-17A, Hs00174383_m1; (human) 18S, Hs03003631_g1 and IFNG, Hs00989291_m1.

Human $C D 4^{+} T$ cell isolation for Western blot and immunofluorescence. $\mathrm{CD}^{+} \mathrm{T}$ cells were purified from PBMCs with the CD4 ${ }^{+} \mathrm{T}$ Cells Isolation Kit (Miltenyi Biotec). Lysates were separated using a NuPAGE 4-12\% Bis-Tris Protein Gel and transferred onto a nitrocellulose membrane. After blocking with 10\% BSA for 30 minutes at RT, the membrane was incubated with a PAD2-specific primary antibody (Proteintech Group) overnight at $4^{\circ} \mathrm{C}$. The membrane was washed 3 times with PBS-Tween $(0.1 \%)$ and incubated with a secondary antibody coupled to IRDye $800 \mathrm{CW}$. After additional washing, the membrane was developed using a Li-COR Odyssey Clx scanner. A subset of sorted cells was seeded onto coverslips coated with poly-L-lysine and fixed with $4 \%$ paraformaldehyde in PBS. Fixed cells were permeabilized with $0.1 \%$ Triton X-100 (MilliporeSigma), and nonspecific binding was blocked with 10\% normal goat serum (NGS; Thermo FIsher Scientific) for 30 minutes at RT. Cells were stained in 1\% NGS with the aforementioned PAD2-specific antibody for 1 hour at RT. After washing, the cells were stained with a secondary antibody conjugated with Alexa Fluor 488 for 30 minutes at RT. Hoechst-stained coverslips were then mounted onto glass slides with ProLong Gold Antifade Mountant and cured overnight before imaging.

Treatment of human $C D 4^{+} T$ cells with PAD inhibitors. Purified $\mathrm{CD}^{+} \mathrm{T}$ cells from healthy donors were activated by plate-bound $\alpha$-CD3 $(2 \mu \mathrm{g} / \mathrm{ml})$ and soluble $\alpha$-CD28 $(1 \mu \mathrm{g} / \mathrm{ml})$ (BioLegend) in T cell culture medium (RPMI1640 with 10\% FBS, $2 \mathrm{mM}$ glutamine, penicillin [100 IU/ml], streptomycin $[0.1 \mathrm{mg} / \mathrm{ml}$, $10 \mathrm{mM}$ HEPES, $10 \mathrm{mM}$ sodium pyruvate, and $50 \mu \mathrm{M} \beta$-mercaptoethanol) for 5 days in the presence/ absence of AFM30a (46) (PAD2 inhibitor, $25 \mu \mathrm{M}$ ) and GSK 199 (47) (PAD4 inhibitor, $15 \mu \mathrm{M})$. CD4 ${ }^{+}$T cell activation and apoptosis were determined by CD69 staining and LIVE/DEAD Fixable Aqua Dead Cell Stain Kit (Thermo Fisher Scientific).

Human $T$ cell in vitro polarization. Experiments were performed as described (60). Healthy donor $\mathrm{CD}^{+} \mathrm{T}$ cells were stained with the following antibodies before sorting using the FACSAria IIIu: antiCD4-BV421, -CD45RA-PerCP-Cy5.5, and -CD45RA-APC-Cy7 and sorted for naive T cells $\left(\mathrm{CD} 4{ }^{+} \mathrm{C}-\right.$

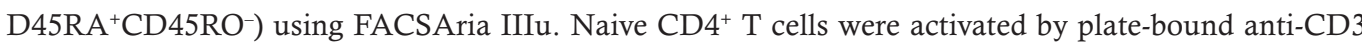
$(2 \mu \mathrm{g} / \mathrm{ml})$ and soluble anti-CD28 $(1 \mu \mathrm{g} / \mathrm{ml})$ (BioLegend) in T cell culture medium (RPMI1640 [Thermo Fisher Scientific] with 10\% FBS, $2 \mathrm{mM}$ glutamine, penicillin (100 IU/ml), streptomycin $(0.1 \mathrm{mg} / \mathrm{ml}), 10$ $\mathrm{mM}$ HEPES, $1 \mathrm{mM} \mathrm{mM}$ sodium pyruvate, and $50 \mu \mathrm{M} \beta$-mercaptoethanol for 7 days in the presence/ absence of various cytokines, antibodies, and PAD inhibitors as indicated. Conditions were as follows: (Th0) anti-IFN- $\gamma(10 \mu \mathrm{g} / \mathrm{ml})$, anti-IL-4 $(5 \mu \mathrm{g} / \mathrm{m})$, anti-IL-10 $(5 \mu \mathrm{g} / \mathrm{ml})$, and IL-2 (100 U/ml); (Th1) IL-12 (10 ng/ml), anti-IL-4 (5 $\mu \mathrm{g} / \mathrm{ml})$, anti-IL-10 (5 $\mu \mathrm{g} / \mathrm{ml})$, and IL-2 (100 U/ml); and (Th17) IL-6 (20 $\mathrm{ng} / \mathrm{ml}), \mathrm{IL}-1 \beta(20 \mathrm{ng} / \mathrm{ml}), \mathrm{IL}-23(50 \mathrm{ng} / \mathrm{ml})$, anti-IFN- $\gamma(10 \mu \mathrm{g} / \mathrm{ml})$, anti-IL-4 $(5 \mu \mathrm{g} / \mathrm{ml})$, and anti-IL-10 $(5 \mu \mathrm{g} / \mathrm{ml})$. PAD inhibitors were AFM30a (PAD2 inhibitor, $25 \mu \mathrm{M})$ and GSK199 (PAD4 inhibitor, 15 $\mu \mathrm{M})$. For intracellular cytokine staining, cells were restimulated for 4 hours with PMA (50 ng/ml) and ionomycin $(750 \mu \mathrm{g} / \mathrm{ml})$, with the addition of Monensin (BD GolgiStop). Cells were stained for CD4 (BV421), CD3 (APC-Cy7), CD45RA ${ }^{+}$(PerCP-Cy5.5), IL-17A (PE), and IFN- $\gamma$ (FITC).

Intracellular staining in human PBMCs. Human PBMCs were isolated as described above, permeabilized with eBioscience Foxp3/Transcription Factor Staining Buffer Set (Thermo Fisher Scientific), blocked with Fc blocker (BioLegend) for 15 minutes, and stained for surface and intracellular markers: CD15 (PE-Cy7), CD14 (FITC), and CD4 (APC-Cy7). Cells were permeabilized and stained with either rabbit isotype control (Invitrogen), PAD2 (Proteintech), or PAD4 (Abcam) for 30 minutes, followed by staining with PE-conjugated donkey anti-rabbit IgG (BioLegend). 
Statistics. Data were plotted and analyzed using the GraphPad Prism 6 software. Statistical significance was calculated by 2-tailed Student's $t$ tests or, for non-Gaussian distributions, using the Mann-Whitney $U$ test. For the vasorelaxation experiments, 2-way ANOVA with post-hoc Tukey's test was used to compare differences between the groups. $P<0.05$ was considered statistically significant.

Study approval. Healthy controls and subjects with a diagnosis of SLE were recruited at the Clinical Center, NIH. All individuals signed informed consent under NIAMS/NIDDK IRB-approved protocol, NIH 94-AR-0066. All mouse experiments were approved by the NIAMS Animal Ethics Committee (ACUC).

\section{Author contributions}

YL and YLL designed and performed experiments, analyzed data, and contributed to writing manuscript; NS performed experiments, analyzed data, and contributed to writing manuscript; CCR performed experiments and analyzed data; EM designed experiments, performed experiments, and analyzed data; LO, RG, PM, KG, KJ, and HWS analyzed gene expression data; VH analyzed histology; SM and PNP synthesized PAD inhibitors; SD performed RNA-Seq; PRT and SAC contributed with crucial reagents generated in their labs and provided critical review of manuscript; and MJK designed the studies, analyzed data, and contributed to writing manuscript.

\section{Acknowledgments}

We thank the Office of Science and Technology, NIAMS/NIH, for technical support. This study utilized the high-performance computational capabilities of the Helix Systems at the NIH (https://hpc.nih.gov/ systems/helix.html). This work was supported by the IRP, NIAMS/NIH (ZIAAR041199), and by the Alliance for Lupus Research.

Address correspondence to: Mariana J. Kaplan, NIAMS/NIH, 10 Center Drive, 12N248C, Bethesda, Maryland 20892, USA. Phone: 301.496.0517; Email: Mariana.kaplan@nih.gov.

1. Tsokos GC. Systemic lupus erythematosus. N Engl J Med. 2011;365(22):2110-2121

2. Crow MK. Type I interferon in the pathogenesis of lupus. J Immunol. 2014;192(12):5459-5468.

3. Kahlenberg JM, Kaplan MJ. Mechanisms of premature atherosclerosis in rheumatoid arthritis and lupus. Annu Rev Med. 2013;64:249-263.

4. Denny MF, et al. A distinct subset of proinflammatory neutrophils isolated from patients with systemic lupus erythematosus induces vascular damage and synthesizes type I IFNs. J Immunol. 2010;184(6):3284-3297.

5. Witalison EE, Thompson PR, Hofseth LJ. Protein Arginine Deiminases and Associated Citrullination: Physiological Functions and Diseases Associated with Dysregulation. Curr Drug Targets. 2015;16(7):700-710.

6. Willemze A, et al. The interaction between HLA shared epitope alleles and smoking and its contribution to autoimmunity against several citrullinated antigens. Arthritis Rheum. 2011;63(7):1823-1832.

7. Carmona-Rivera C, et al. Synovial fibroblast-neutrophil interactions promote pathogenic adaptive immunity in rheumatoid arthritis. Sci Immunol. 2017;2(10):eaag3358.

8. Mastronardi FG, et al. Increased citrullination of histone H3 in multiple sclerosis brain and animal models of demyelination: a role for tumor necrosis factor-induced peptidylarginine deiminase 4 translocation. J Neurosci. 2006;26(44):11387-11396.

9. Stadler SC, et al. Dysregulation of PAD4-mediated citrullination of nuclear GSK3 $\beta$ activates TGF- $\beta$ signaling and induces epithelial-to-mesenchymal transition in breast cancer cells. Proc Natl Acad Sci USA. 2013;110(29):11851-11856.

10. Christophorou MA, et al. Citrullination regulates pluripotency and histone H1 binding to chromatin. Nature. 2014;507(7490):104-108.

11. Cuthbert GL, et al. Histone deimination antagonizes arginine methylation. Cell. 2004;118(5):545-553.

12. Neeli I, Khan SN, Radic M. Histone deimination as a response to inflammatory stimuli in neutrophils. J Immunol. 2008;180(3):1895-1902.

13. Wang $\mathrm{Y}$, et al. Histone hypercitrullination mediates chromatin decondensation and neutrophil extracellular trap formation. J Cell Biol. 2009;184(2):205-213.

14. Rohrbach AS, Slade DJ, Thompson PR, Mowen KA. Activation of PAD4 in NET formation. Front Immunol. 2012;3:360.

15. Brinkmann V, et al. Neutrophil extracellular traps kill bacteria. Science. 2004;303(5663):1532-1535.

16. Corsiero E, et al. Single cell cloning and recombinant monoclonal antibodies generation from RA synovial B cells reveal frequent targeting of citrullinated histones of NETs. Ann Rheum Dis. 2016;75(10):1866-1875.

17. Khandpur R, et al. NETs are a source of citrullinated autoantigens and stimulate inflammatory responses in rheumatoid arthritis. Sci Transl Med. 2013;5(178):178ra40.

18. Lin AM, et al. Mast cells and neutrophils release IL-17 through extracellular trap formation in psoriasis. J Immunol. 2011;187(1):490-500.

19. Kessenbrock K, et al. Netting neutrophils in autoimmune small-vessel vasculitis. Nat Med. 2009;15(6):623-625.

20. Lood C, et al. Neutrophil extracellular traps enriched in oxidized mitochondrial DNA are interferogenic and contribute to lupus-like disease. Nat Med. 2016;22(2):146-153. 
21. Villanueva E, et al. Netting neutrophils induce endothelial damage, infiltrate tissues, and expose immunostimulatory molecules in systemic lupus erythematosus. J Immunol. 2011;187(1):538-552.

22. Lande R, et al. Neutrophils activate plasmacytoid dendritic cells by releasing self-DNA-peptide complexes in systemic lupus erythematosus. Sci Transl Med. 2011;3(73):73ra19.

23. Hakkim A, et al. Impairment of neutrophil extracellular trap degradation is associated with lupus nephritis. Proc Natl Acad Sci USA. 2010;107(21):9813-9818.

24. Kahlenberg JM, Carmona-Rivera C, Smith CK, Kaplan MJ. Neutrophil extracellular trap-associated protein activation of the NLRP3 inflammasome is enhanced in lupus macrophages. J Immunol. 2013;190(3):1217-1226.

25. Carmona-Rivera C, Zhao W, Yalavarthi S, Kaplan MJ. Neutrophil extracellular traps induce endothelial dysfunction in systemic lupus erythematosus through the activation of matrix metalloproteinase-2. Ann Rheum Dis. 2015;74(7):1417-1424.

26. Sun B, et al. Citrullination of NF-kB p65 promotes its nuclear localization and TLR-induced expression of IL-1 $\beta$ and TNF $\alpha$. Sci Immunol. 2017;2:eaal3062.

27. Vossenaar ER, et al. Citrullination of synovial proteins in murine models of rheumatoid arthritis. Arthritis Rheum. 2003;48(9):2489-2500

28. Zhou Y, et al. Spontaneous Secretion of the Citrullination Enzyme PAD2 and Cell Surface Exposure of PAD4 by Neutrophils. Front Immunol. 2017;8:1200.

29. Bawadekar M, et al. Peptidylarginine deiminase 2 is required for tumor necrosis factor alpha-induced citrullination and arthritis but not neutrophil extracellular trap formation. J Autoimmun. 2017;80:39-47.

30. Chang HH, et al. A molecular signature of preclinical rheumatoid arthritis triggered by dysregulated PTPN22. JCI Insight. 2016;1(17):e90045.

31. Knight JS, et al. Peptidylarginine deiminase inhibition is immunomodulatory and vasculoprotective in murine lupus. J Clin Invest. 2013;123(7):2981-2993

32. Knight JS, et al. Peptidylarginine deiminase inhibition disrupts NET formation and protects against kidney, skin and vascular disease in lupus-prone MRL/lpr mice. Ann Rheum Dis. 2015;74(12):2199-2206.

33. Knight JS, et al. Peptidylarginine deiminase inhibition reduces vascular damage and modulates innate immune responses in murine models of atherosclerosis. Circ Res. 2014;114(6):947-956.

34. Gordon RA, et al. Lupus and proliferative nephritis are PAD4 independent in murine models. JCI Insight. 2017;2(10):e92926.

35. Kienhöfer D, et al. Experimental lupus is aggravated in mouse strains with impaired induction of neutrophil extracellular traps. JCI Insight. 2017;2(10):e92920.

36. Loos T, et al. Citrullination of CXCL10 and CXCL11 by peptidylarginine deiminase: a naturally occurring posttranslational modification of chemokines and new dimension of immunoregulation. Blood. 2008;112(7):2648-2656.

37. Liu Y, Seto NL, Carmona-Rivera C, Kaplan MJ. Accelerated model of lupus autoimmunity and vasculopathy driven by toll-like receptor 7/9 imbalance. Lupus Sci Med. 2018;5(1):e000259.

38. Souyris M, et al. TLR7 escapes X chromosome inactivation in immune cells. Sci Immunol. 2018;3(19):eaap8855.

39. Das A, et al. Follicular Dendritic Cell Activation by TLR Ligands Promotes Autoreactive B Cell Responses. Immunity. 2017;46(1):106-119.

40. Pisitkun P, Deane JA, Difilippantonio MJ, Tarasenko T, Satterthwaite AB, Bolland S. Autoreactive B cell responses to RNA-related antigens due to TLR7 gene duplication. Science. 2006;312(5780):1669-1672.

41. Yokogawa M, et al. Epicutaneous application of toll-like receptor 7 agonists leads to systemic autoimmunity in wild-type mice: a new model of systemic Lupus erythematosus. Arthritis Rheumatol. 2014;66(3):694-706.

42. Warnatsch A, Ioannou M, Wang Q, Papayannopoulos V. Inflammation. Neutrophil extracellular traps license macrophages for cytokine production in atherosclerosis. Science. 2015;349(6245):316-320.

43. Liu Y, et al. Myeloid-Specific Deletion of Peptidylarginine Deiminase 4 Mitigates Atherosclerosis. Front Immunol. 2018;9:1680.

44. Zhang X, et al. Peptidylarginine deiminase 2-catalyzed histone $\mathrm{H} 3$ arginine 26 citrullination facilitates estrogen receptor $\alpha$ target gene activation. Proc Natl Acad Sci USA. 2012;109(33):13331-13336.

45. Vossenaar ER, et al. Expression and activity of citrullinating peptidylarginine deiminase enzymes in monocytes and macrophages. Ann Rheum Dis. 2004;63(4):373-381.

46. Muth A, et al. Development of a Selective Inhibitor of Protein Arginine Deiminase 2. J Med Chem. 2017;60(7):3198-3211

47. Lewis HD, et al. Inhibition of PAD4 activity is sufficient to disrupt mouse and human NET formation. Nat Chem Biol. 2015;11(3):189-191.

48. Meegan JE, et al. Citrullinated histone 3 causes endothelial barrier dysfunction. Biochem Biophys Res Commun. 2018;503(3):1498-1502

49. Fatenejad S, Peng SL, Disorbo O, Craft J. Central T cell tolerance in lupus-prone mice: influence of autoimmune background and the lpr mutation. J Immunol. 1998;161(11):6427-6432.

50. Kambham N. Crescentic Glomerulonephritis: an update on Pauci-immune and Anti-GBM diseases. Adv Anat Pathol. 2012;19(2):111-124.

51. Rudofsky UH. Murine lupus nephritis is accelerated by anti-glomerular basement membrane autoantibodies. Clin Exp Immunol. $1981 ; 44(1): 18-23$

52. Bagavant H, Fu SM. Pathogenesis of kidney disease in systemic lupus erythematosus. Curr Opin Rheumatol. 2009;21(5):489-494.

53. Schauer C, et al. Aggregated neutrophil extracellular traps limit inflammation by degrading cytokines and chemokines. Nat Med. 2014;20(5):511-517.

54. Seri Y, et al. Peptidylarginine deiminase type 4 deficiency reduced arthritis severity in a glucose-6-phosphate isomerase-induced arthritis model. Sci Rep. 2015;5:13041.

55. Moon JS, et al. Intranuclear delivery of the transcription modulation domain of Tbet-improved lupus nephritis in (NZB/NZW) F1 lupus-prone mice. Kidney Int. 2018;93(5):1118-1130.

56. Ooi JD, Kitching AR. CD4+ Th1 cells are effectors in lupus nephritis--but what are their targets? Kidney Int. 2012;82(9):947-949.

57. Hochberg MC. Updating the American College of Rheumatology revised criteria for the classification of systemic lupus erythematosus. Arthritis Rheum. 1997;40(9):1725. 
58. Bombardier C, Gladman DD, Urowitz MB, Caron D, Chang CH. Derivation of the SLEDAI. A disease activity index for lupus patients. The Committee on Prognosis Studies in SLE. Arthritis Rheum. 1992;35(6):630-640.

59. Furumoto Y, et al. Tofacitinib Ameliorates Murine Lupus and Its Associated Vascular Dysfunction. Arthritis Rheumatol. 2017;69(1):148-160

60. Poholek AC, et al. IL-10 induces a STAT3-dependent autoregulatory loop in TH2 cells that promotes Blimp-1 restriction of cell expansion via antagonism of STAT5 target genes. Sci Immunol. 2016;1(5):eaaf8612.

61. Kim D, Pertea G, Trapnell C, Pimentel H, Kelley R, Salzberg SL. TopHat2: accurate alignment of transcriptomes in the presence of insertions, deletions and gene fusions. Genome Biol. 2013;14(4):R36.

62. Yu G, Wang LG, Han Y, He QY. clusterProfiler: an R package for comparing biological themes among gene clusters. OMICS. 2012;16(5):284-287. 